\title{
Effects of new heavy fermions on complex scalar dark matter phenomenology in gauged two Higgs doublet model
}

\author{
Bayu Dirgantara ${ }^{1, \mathrm{a}}{ }_{\mathbb{D}}$, Chrisna Setyo Nugroho $^{2, \mathrm{~b}}{ }_{(\mathbb{D})}$ \\ ${ }^{1}$ School of Physics and Center of Excellence in High Energy Physics and Astrophysics, Suranaree University of Technology, Nakhon Ratchasima \\ 30000, Thailand \\ ${ }^{2}$ Physics Division, National Center for Theoretical Sciences, National Tsing-Hua University, Hsinchu 30013, Taiwan
}

Received: 5 July 2021 / Accepted: 25 January 2022 / Published online: 15 February 2022

(C) The Author(s) 2022

\begin{abstract}
We study the inclusion of new heavy fermions on complex scalar dark matter (DM) phenomenology within gauged two Higgs doublet model (G2HDM). We find that for DM mass above $1 \mathrm{TeV}$, heavy quarks coannihilations into the Standard Model (SM) quarks and gluons dominate the thermally-averaged cross section relevant for the relic abundance of complex scalar DM. We demonstrate that the effects of QCD Sommerfeld correction as well as QCD bound state formation in determining the DM relic density are negligible. We show that the allowed parameter space is significantly constrained by the current PLANCK relic density data as well as XENON1T limit appropriate for DM direct search.
\end{abstract}

\section{Introduction}

The nature of dark matter (DM) is one of the open problems in cosmology, astrophysics and particle physics. Apart from its gravitational interaction, there is no evidence that it interacts with ordinary matter via the other existing forces in nature. Furthermore, the current understanding of our universe can be explained very well if one includes the existence of the cold DM in addition to the ordinary matter and dark energy. It has been assumed that the DM was in thermal equilibrium with the Standard Model (SM) particles in the early universe. As the reaction rate that keeps the DM in thermal equilibrium with other SM particles drops out and becomes comparable with the expansion rate of the universe, it froze out. As a result, it remains with us today. One of the most popular and well studied DM candidates so far is the weakly interacting massive particle (WIMP). This candidate fits the observed DM abundance with the typical electroweak scale annihilation cross section.

\footnotetext{
a e-mail: bayuquarkquantum@yahoo.com (corresponding author)

b e-mail: setyo13nugros@gmail.com
}

The need to extend the SM particle contents is necessary since it does not provide any suitable DM candidate. One of the most studied examples of the SM extension is the general two Higgs doublet model (2HDM). This model comes with many variations and each of them offers different phenomenological features, see for example $[1,2]$ for a review. As one of the 2HDM variants, the inert Higgs doublet model (IHDM) [3] provides a suitable DM candidate. This is done by imposing the discrete $\mathcal{Z}_{2}$ symmetry in which the second Higgs doublet belongs to the $\mathcal{Z}_{2}$-odd particle. This model has been studied in detail over the years [4-30]. Recently, this $\mathcal{Z}_{2}$ symmetry appears as an accidental symmetry in a renormalizable gauged two Higgs doublet model (G2HDM) and it has been studied in detail in [31,32]. In this model, the two Higgs doublets are put together in a doublet representation of an additional $S U(2)_{H}$ gauge group. In addition to this, there is also a new $U(1)_{X}$ symmetry. As for the scalar sector, it is expanded by including a new $S U(2)_{H}$ triplet and doublet. These new scalars transform trivially under the SM gauge group.

As in IHDM, there is a suitable scalar DM candidate in G2HDM. In this case, the stability of the DM is protected by the accidental $\mathcal{Z}_{2}$ symmetry. The phenomenological study of this DM has been done in [32]. This study has taken the previous results of the G2HDM studies [33-37] into account, especially the results from $[36,37]$. These two studies put constraints in the scalar sector and gauge sector of the G2HDM. These include vacuum stability of the scalar potential, perturbative unitarity, Higgs physics, Drell-Yan process, the $Z^{\prime}$ search, as well as electroweak precision test (EWPT). We dubbed these results as the scalar and gauge sector constraints (SGSC). However, the previous DM study in [32] neglected the heavy $\mathcal{Z}_{2}$-odd fermions in their calculation. In this paper, we focus on the effects of these new heavy fermions on complex scalar DM phenomenology. For consistency with the 
previous DM study, we take SGSC constraints as our starting point.

This paper is organized as follows. In Sect. 2 we briefly mention the important aspects of the G2HDM model, especially the scalar potential, mass spectra, and suitable dark matter candidates. To compare our DM study against the existing experimental data, we discuss how relic density (RD) and direct detection (DD) constrain our complex scalar DM. This is discussed in Sect. 3. In Sect. 4, we briefly explain the methodology employed in our numerical computation. We further discuss in detail the results obtained in our analysis. In Sect. 5, we discuss QCD Sommerfeld correction and QCD bound state effect relevant for the relic density calculation. The allowed parameter space after imposing DM physics constraints is discussed in Sect. 6. Finally, we summarize and conclude our study in Sect. 7. We list the most important Feynman rules used in the discussion of this work in Appendix A.

\section{The G2HDM model}

\subsection{Matter content}

The gauge group of G2HDM is realized by expanding the SM gauge symmetry with additional $S U(2)_{H} \times U(1)_{X}$ dubbed as hidden gauge sector. The SM scalar sector $H_{1}$ is extended by adding one Higgs doublet $H_{2}$ in such a way that both of them transform under the fundamental representation of $S U(2)_{L}$ and $S U(2)_{H}$ gauge group. Furthermore, $\Delta_{H}$ and $\Phi_{H}$, which transform under the triplet and doublet representations of $S U(2)_{H}$, are present. Both of them are SM singlets.

New right-handed heavy fermions are put together with the SM right-handed fermions into $S U(2)_{H}$ doublets while maintaining the trivial representation of $S U(2)_{L}$. Furthermore, anomaly cancellation dictates us to further include two pairs of left-handed heavy quarks and two pairs of left-handed heavy leptons for each generation, which are singlets under $S U(2)_{L}$ and $U(1)_{X}$. As a remark, one notes that the $S U(2)_{H}$ considered here is different from the $S U(2)_{R}$ in left-right symmetric models $[38,39]$. The $W^{\prime(p, m)}$ in G2HDM is electrically neutral, while the $W^{\prime \pm}$ in left-right symmetric carry non-zero electric charges. This is the rationale behind the superscripts $p$ and $m$ in labelling $W^{\prime}$. As another comparison, we also observe that non-sterile right-handed neutrinos $v_{l R} \mathrm{~S}$, incorporated in the mirror fermion models of electroweak scale right-handed neutrinos [40-45], are different from our setup. In the mirror fermion models, $\nu_{l R} \mathrm{~s}$ are paired together with mirror charged leptons $l_{R}^{M}$ s to form $S U(2)_{L}$ doublets. In contrast, they are grouped with new heavy right-handed neutrinos $v_{l R}^{H}$ to form $S U(2)_{H}$ doublets in G2HDM. There are several models that impose additional gauge symmetry on 2HDM to solve flavor problem, dark matter and neutrino masses, see for instance [46-51]. In Table 1, we list the matter contents of the G2HDM model and their associated quantum numbers.

\subsection{Scalar potential and mass spectra}

\section{Scalar potential}

The most general renormalizable scalar potential that satisfies the G2HDM symmetries comprises 4 distinct terms [36]

$V_{T}=V(H)+V\left(\Phi_{H}\right)+V\left(\Delta_{H}\right)+V_{\text {mix }}\left(H, \Delta_{H}, \Phi_{H}\right)$.

The first term $V(H)$ in Eq. (1) contains self-interaction of $S U(2)_{L}$ and $S U(2)_{H}$ scalar doublet $H$ reads as

$$
\begin{aligned}
V(H)= & \mu_{H}^{2}\left(H^{\alpha i} H_{\alpha i}\right)+\lambda_{H}\left(H^{\alpha i} H_{\alpha i}\right)^{2} \\
& +\frac{1}{2} \lambda_{H}^{\prime} \epsilon_{\alpha \beta} \epsilon^{\gamma \delta}\left(H^{\alpha i} H_{\gamma i}\right)\left(H^{\beta j} H_{\delta j}\right) \\
= & \mu_{H}^{2}\left(H_{1}^{\dagger} H_{1}+H_{2}^{\dagger} H_{2}\right)+\lambda_{H}\left(H_{1}^{\dagger} H_{1}+H_{2}^{\dagger} H_{2}\right)^{2} \\
& +\lambda_{H}^{\prime}\left(-H_{1}^{\dagger} H_{1} H_{2}^{\dagger} H_{2}+H_{1}^{\dagger} H_{2} H_{2}^{\dagger} H_{1}\right)
\end{aligned}
$$

where Greek and Latin letters indicate $S U(2)_{H}$ and $S U(2)_{L}$ indices respectively, both of which run from 1 to 2 , and the upper and lower indices are related by complex conjugation, i.e., $H^{\alpha i}=H_{\alpha i}^{*}$. A close inspection shows that the second line of Eq. (2) exhibits the discrete $\mathcal{Z}_{2}$ symmetry of $H_{1} \rightarrow H_{1}$ and $\mathrm{H}_{2} \rightarrow-\mathrm{H}_{2}$. The appearance of this discrete symmetry in G2HDM model is more natural unlike the discrete symmetry in general $2 \mathrm{HDM}$ model. In the latter case, one needs to put this symmetry by hand to forbid FCNC at tree level in the Yukawa sectors. The second term $V\left(\Phi_{H}\right)$ is the selfinteraction of $\Phi_{H}$

$$
\begin{aligned}
V\left(\Phi_{H}\right) & =\mu_{\Phi}^{2} \Phi_{H}^{\dagger} \Phi_{H}+\lambda_{\Phi}\left(\Phi_{H}^{\dagger} \Phi_{H}\right)^{2} \\
& =\mu_{\Phi}^{2}\left(\Phi_{1}^{*} \Phi_{1}+\Phi_{2}^{*} \Phi_{2}\right)+\lambda_{\Phi}\left(\Phi_{1}^{*} \Phi_{1}+\Phi_{2}^{*} \Phi_{2}\right)^{2}
\end{aligned}
$$

where $\Phi_{H}=\left(\Phi_{1}, \Phi_{2}\right)^{\mathrm{T}}$ belongs to the $S U(2)_{H}$ doublet. The self-interaction of the $S U(2)_{H}$ scalar triplet $\Delta_{H}$ reads

$$
\begin{aligned}
V\left(\Delta_{H}\right)= & -\mu_{\Delta}^{2} \operatorname{Tr}\left(\Delta_{H}^{2}\right)+\lambda_{\Delta}\left(\operatorname{Tr}\left(\Delta_{H}^{2}\right)\right)^{2} \\
= & -\mu_{\Delta}^{2}\left(\frac{1}{2} \Delta_{3}^{2}+\Delta_{p} \Delta_{m}\right) \\
& +\lambda_{\Delta}\left(\frac{1}{2} \Delta_{3}^{2}+\Delta_{p} \Delta_{m}\right)^{2}
\end{aligned}
$$


where the triplet field is expressed as

$$
\begin{aligned}
\Delta_{H} & =\left(\begin{array}{cc}
\Delta_{3} / 2 & \Delta_{p} / \sqrt{2} \\
\Delta_{m} / \sqrt{2} & -\Delta_{3} / 2
\end{array}\right)=\Delta_{H}^{\dagger} \text { with } \\
\Delta_{m} & =\left(\Delta_{p}\right)^{*} \text { and }\left(\Delta_{3}\right)^{*}=\Delta_{3} .
\end{aligned}
$$

Here, the off-diagonal components of the $S U(2)_{H}$ triplet, $\Delta_{H}$, are electrically neutral. Following the same labelling as in $W^{\prime(p, m)}$, we put the subscripts $p$ and $m$ on them. Finally, the last term $V_{\text {mix }}$ takes all possible mixing between $H$ and $\Phi_{H}$ as well as $\Delta_{H}$ into account, and it is given by

$$
\begin{aligned}
V_{\text {mix }}\left(H, \Delta_{H}, \Phi_{H}\right)= & +M_{H \Delta}\left(H^{\dagger} \Delta_{H} H\right) \\
& -M_{\Phi \Delta}\left(\Phi_{H}^{\dagger} \Delta_{H} \Phi_{H}\right) \\
& +\lambda_{H \Phi}\left(H^{\dagger} H\right)\left(\Phi_{H}^{\dagger} \Phi_{H}\right) \\
& +\lambda_{H \Phi}^{\prime}\left(H^{\dagger} \Phi_{H}\right)\left(\Phi_{H}^{\dagger} H\right) \\
& +\lambda_{H \Delta}\left(H^{\dagger} H\right) \operatorname{Tr}\left(\Delta_{H}^{2}\right) \\
& +\lambda_{\Phi \Delta}\left(\Phi_{H}^{\dagger} \Phi_{H}\right) \operatorname{Tr}\left(\Delta_{H}^{2}\right) .
\end{aligned}
$$

The explicit expression of Eq. (6) in terms of its components is

$$
\begin{aligned}
V_{\text {mix }} & \left(H, \Delta_{H}, \Phi_{H}\right) \\
= & +M_{H \Delta}\left(\frac{1}{\sqrt{2}} H_{1}^{\dagger} H_{2} \Delta_{p}+\frac{1}{2} H_{1}^{\dagger} H_{1} \Delta_{3}\right. \\
& \left.+\frac{1}{\sqrt{2}} H_{2}^{\dagger} H_{1} \Delta_{m}-\frac{1}{2} H_{2}^{\dagger} H_{2} \Delta_{3}\right)-M_{\Phi \Delta} \\
& \times\left(\frac{1}{\sqrt{2}} \Phi_{1}^{*} \Phi_{2} \Delta_{p}+\frac{1}{2} \Phi_{1}^{*} \Phi_{1} \Delta_{3}\right. \\
& \left.+\frac{1}{\sqrt{2}} \Phi_{2}^{*} \Phi_{1} \Delta_{m}-\frac{1}{2} \Phi_{2}^{*} \Phi_{2} \Delta_{3}\right)+\lambda_{H \Phi} \\
& \times\left(H_{1}^{\dagger} H_{1}+H_{2}^{\dagger} H_{2}\right)\left(\Phi_{1}^{*} \Phi_{1}+\Phi_{2}^{*} \Phi_{2}\right) \\
& +\lambda_{H \Phi}^{\prime}\left(H_{1}^{\dagger} H_{1} \Phi_{1}^{*} \Phi_{1}+H_{2}^{\dagger} H_{2} \Phi_{2}^{*} \Phi_{2}\right. \\
& \left.+H_{1}^{\dagger} H_{2} \Phi_{2}^{*} \Phi_{1}+H_{2}^{\dagger} H_{1} \Phi_{1}^{*} \Phi_{2}\right) \\
& +\lambda_{H \Delta}\left(H_{1}^{\dagger} H_{1}+H_{2}^{\dagger} H_{2}\right) \\
& \times\left(\frac{1}{2} \Delta_{3}^{2}+\Delta_{p} \Delta_{m}\right)+\lambda_{\Phi \Delta} \\
& \times\left(\Phi_{1}^{*} \Phi_{1}+\Phi_{2}^{*} \Phi_{2}\right)\left(\frac{1}{2} \Delta_{3}^{2}+\Delta_{p} \Delta_{m}\right) .
\end{aligned}
$$

As before, $V_{\text {mix }}\left(H, \Delta_{H}, \Phi_{H}\right)$ is also invariant under $H_{1} \rightarrow H_{1}, H_{2} \rightarrow-H_{2}, \Phi_{1} \rightarrow-\Phi_{1}, \Phi_{2} \rightarrow \Phi_{2}, \Delta_{3} \rightarrow \Delta_{3}$, and $\Delta_{p, m} \rightarrow-\Delta_{p, m}$. It has been shown in [32] that this discrete symmetry holds in all sector in G2HDM.
Table 1 Matter contents and their corresponding quantum numbers in G2HDM. New heavy fermions are denoted by the superscript $H$

\begin{tabular}{ll}
\hline Matter fields & $S U(3)_{C} \times S U(2)_{L} \times S U(2)_{H} \times U(1)_{Y} \times U(1)_{X}$ \\
\hline$H=\left(H_{1}, H_{2}\right)^{\mathrm{T}}$ & $(1,2,2,1 / 2,1)$ \\
$\Delta_{H}$ & $(1,1,3,0,0)$ \\
$\Phi_{H}$ & $(1,1,2,0,1)$ \\
$Q_{L}=\left(u_{L}, d_{L}\right)^{\mathrm{T}}$ & $(3,2,1,1 / 6,0)$ \\
$U_{R}=\left(u_{R}, u_{R}^{H}\right)^{\mathrm{T}}$ & $(3,1,2,2 / 3,1)$ \\
$D_{R}=\left(d_{R}^{H}, d_{R}\right)^{\mathrm{T}}$ & $(3,1,2,-1 / 3,-1)$ \\
$L_{L}=\left(v_{L}, e_{L}\right)^{\mathrm{T}}$ & $(1,2,1,-1 / 2,0)$ \\
$N_{R}=\left(v_{R}, v_{R}^{H}\right)^{\mathrm{T}}$ & $(1,1,2,0,1)$ \\
$E_{R}=\left(e_{R}^{H}, e_{R}\right)^{\mathrm{T}}$ & $(1,1,2,-1,-1)$ \\
$v_{L}^{H}$ & $(1,1,1,0,0)$ \\
$e_{L}^{H}$ & $(1,1,1,-1,0)$ \\
$u_{L}^{H}$ & $(3,1,1,2 / 3,0)$ \\
$d_{L}^{H}$ & $(3,1,1,-1 / 3,0)$ \\
\hline
\end{tabular}

\subsection{Mass spectra}

The gauge symmetry of G2HDM is broken spontaneously by the vacuum expectation values (VEVs) of $\left\langle H_{1}\right\rangle=$ $(0, v / \sqrt{2})^{\mathrm{T}},\left\langle\Phi_{2}\right\rangle=v_{\Phi} / \sqrt{2}$, and $\left\langle\Delta_{3}\right\rangle=-v_{\Delta}[31,36]$. As a result of this spontaneous symmetry breaking (SSB), all fields in G2HDM acquire their mass from these VEVs. The mass terms as well as the mixing terms of the scalar fields can be extracted by writing the scalar potential in terms of the existing VEVs and further taking the second derivatives with respect to the corresponding scalar fields. In total there are six massive scalars and massless Goldstone bosons. In the gauge sector, there are seven massive gauge particles.

The SM Higgs can be obtained from the mixing of three real scalars $h, \phi_{2}$ and $\delta_{3} .{ }^{1}$ From this mixing, one obtains three massive eigenvalues. The remaining $\mathcal{Z}_{2}$-even scalars $G^{ \pm, 0}$ and $G_{H}^{0}$ do not mix with the existing scalar fields. These would-be Goldstone bosons will be absorbed by the gauge bosons. Moving to the Dark ( $\mathcal{Z}_{2}$-odd) scalars, one has charged Higgs and three neutral complex fields $G_{H}^{p, m}, H_{2}^{0 *}$ and $\Delta_{p, m}$ that mix with each other. There will be one massless eigenvalue corresponding to the Goldstone boson. The complex gauge bosons $S U(2)_{H}, W^{\prime(p, m)}$, become massive by absorbing this Goldstone particle. Since in this work we consider the complex scalar dark matter, $D$, it is instructive

\footnotetext{
${ }^{1}$ We parameterize the scalar fields as the notations of [31]: $H_{1}=$ $\left(\begin{array}{c}G^{+} \\ \frac{v+h}{\sqrt{2}}+i G^{0}\end{array}\right), H_{2}=\left(\begin{array}{c}H^{+} \\ H_{2}^{0}\end{array}\right), \Phi_{H}=\left(\begin{array}{c}G_{H}^{p} \\ \frac{v_{\Phi}+\phi_{2}}{\sqrt{2}}+i G_{H}^{0}\end{array}\right)$, and $\Delta_{H}=$ $\left(\begin{array}{cc}\frac{-v_{\Delta}+\delta_{3}}{2} & \frac{\Delta_{p}}{\sqrt{2}} \\ \frac{\Delta_{m}}{\sqrt{2}} & \frac{v_{\Delta}-\delta_{3}}{2}\end{array}\right)$.
} 
to show the corresponding mass matrix as follows

$$
\mathcal{M}_{0}^{\prime 2}=\left(\begin{array}{ccc}
M_{\Phi \Delta} v_{\Delta}+\frac{1}{2} \lambda_{H \Phi}^{\prime} v^{2} & \frac{1}{2} \lambda_{H \Phi}^{\prime} v v_{\Phi} & -\frac{1}{2} M_{\Phi \Delta} v_{\Phi} \\
\frac{1}{2} \lambda_{H \Phi}^{\prime} v v_{\Phi} & M_{H \Delta} v_{\Delta}+\frac{1}{2} \lambda_{H \Phi}^{\prime} v_{\Phi}^{2} & \frac{1}{2} M_{H \Delta} v \\
-\frac{1}{2} M_{\Phi \Delta} v_{\Phi} & \frac{1}{2} M_{H \Delta} v & \frac{1}{4 v_{\Delta}}\left(M_{H \Delta} v^{2}+M_{\Phi \Delta} v_{\Phi}^{2}\right)
\end{array}\right)
$$

where this matrix is written in the basis of $\mathcal{G}=\left\{G_{H}^{p}, H_{2}^{0 *}, \Delta_{p}\right\}^{T}$. Upon diagonalization, the interaction basis and mass basis are related via orthogonal matrix $\mathcal{O}^{D}$ as $\mathcal{G}=\mathcal{O}^{D}$. $\left\{\widetilde{G}^{p}, D, \widetilde{\Delta}\right\}^{\mathrm{T}}$ such that the orthogonal matrix $\mathcal{O}^{D}$ brings the mixing matrix into the diagonal form

$\left(\mathcal{O}^{D}\right)^{\mathrm{T}} \cdot \mathcal{M}_{0}^{\prime 2} \cdot \mathcal{O}^{D}=\operatorname{Diag}\left(0, m_{D}^{2}, m_{\widetilde{\Delta}}^{2}\right)$,

where $m_{D}$ and $m_{\tilde{\Delta}}$ denote dark matter mass and heavier dark scalar mass, respectively.

\section{Fermions}

It has been shown in [32] that the $\mathcal{Z}_{2}$ symmetry can be extended in Yukawa sector. Under this symmetry, new heavy fermions $f^{H}$ transform under odd parity while the SM fermions are $\mathcal{Z}_{2}$ even (see also Table 2 below). Depending on the mass splitting as well as the interaction between $f^{H}$ and dark matter, $D$, the heavy fermions could play an important role in determining the DM relic abundance. Thus, it is important to discuss the Yukawa sector of this model.

Let us write down the most general Yukawa interactions in G2HDM model and the corresponding fermionic mass terms. Starting from the quark sector, we set the $S U(2)_{L}$ quark doublet, $Q_{L}$, to be an $S U(2)_{H}$ singlet and include additional $S U(2)_{L}$ singlets $u_{R}^{H}$ and $d_{R}^{H}$ which together with the SM right-handed quarks $u_{R}$ and $d_{R}$, respectively, to form $S U(2)_{H}$ doublets consistent with Table 1, i.e., $U_{R}^{T}=$

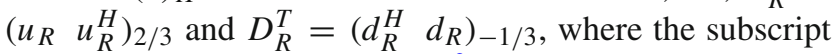
represents hypercharge, we have ${ }^{2}$

$$
\begin{aligned}
\mathcal{L}_{\text {Yuk }} & \supset y_{d} \bar{Q}_{L}\left(D_{R} \cdot H\right)+y_{u} \bar{Q}_{L}\left(U_{R} \cdot \widetilde{\widetilde{H}}\right)+\text { H.c. } \\
= & y_{d} \bar{Q}_{L}\left(d_{R}^{H} H_{2}-d_{R} H_{1}\right) \\
& -y_{u} \bar{Q}_{L}\left(u{ }_{R} \tilde{H}_{1}+u_{R}^{H} \tilde{H}_{2}\right)+\text { H.c. }
\end{aligned}
$$

where $\widetilde{\widetilde{H}} \equiv\left(\tilde{H}_{2}-\tilde{H}_{1}\right)^{T}$ with $\tilde{H}_{1,2}=i \tau_{2} H_{1,2}^{*}$. After the EW symmetry breaking $\left\langle H_{1}\right\rangle \neq 0, u$ and $d$ obtain their masses but $u_{R}^{H}$ and $d_{R}^{H}$ remain massless since $H_{2}$ has no VEV.

\footnotetext{
$\overline{2} A \cdot B$ is defined as $\epsilon_{i j} A^{i} B^{j}$ where $A$ and $B$ are two 2-dimensional spinor representations of $S U(2)_{H}$.
}

To give masses to the new fermions, we employ the $S U(2)_{H}$ scalar doublet $\Phi_{H}=\left(\Phi_{1} \Phi_{2}\right)^{T}$, which is singlet under $S U(2)_{L}$, to write down the following Yukawa interactions for the left-handed $S U(2)_{L, H}$ singlets $u_{L}^{H}$ and $d_{L}^{H}$ and the $S U(2)_{H}$ doublets $U_{R}$ and $D_{R}$,

$$
\begin{aligned}
\mathcal{L}_{\text {Yuk }} \supset & -y_{d}^{\prime} \bar{d}_{L}^{H}\left(D_{R} \cdot \Phi_{H}\right)+y_{u}^{\prime} \bar{u}_{L}^{H}\left(U_{R} \cdot \tilde{\Phi}_{H}\right)+\text { H.c. } \\
= & -y_{d}^{\prime} \bar{d}_{L}^{H}\left(d_{R}^{H} \Phi_{2}-d_{R} \Phi_{1}\right) \\
& -y_{u}^{\prime} \bar{u}_{L}^{H}\left(u_{R} \Phi_{1}^{*}+u_{R}^{H} \Phi_{2}^{*}\right)+\text { H.c. }
\end{aligned}
$$

where $\Phi$ has $Y=0, Y\left(u_{L}^{H}\right)=Y\left(U_{R}\right)=2 / 3$ and $Y\left(d_{L}^{H}\right)=$ $Y\left(D_{R}\right)=-1 / 3$ Since $\tilde{\Phi}_{H}=\left(\Phi_{2}^{*}-\Phi_{1}^{*}\right)^{T}$. Since $\left\langle\Phi_{2}\right\rangle=$ $v_{\Phi} / \sqrt{2}, u_{R}^{H}\left(u_{L}^{H}\right)$ and $d_{R}^{H}\left(d_{L}^{H}\right)$ masses are $y_{u}^{\prime} v_{\Phi} / \sqrt{2}$ and $y_{d}^{\prime} v_{\Phi} / \sqrt{2}$, respectively. Note that there is no contribution from $v_{\Delta}$ for the masses for both the SM and new fermions.

The lepton sector is similar to the quark sector and it is given by

$$
\begin{aligned}
\mathcal{L}_{\mathrm{Yuk}} \supset & y_{e} \bar{L}_{L}\left(E_{R} \cdot H\right)+y_{v} \bar{L}_{L}\left(N_{R} \cdot \widetilde{\widetilde{H}}\right) \\
& -y_{e}^{\prime} \bar{e}_{L}^{H}\left(E_{R} \cdot \Phi_{H}\right)+y_{v}^{\prime} \bar{v}_{L}^{H}\left(N_{R} \cdot \tilde{\Phi}_{H}\right)+\text { H.c. }, \\
= & y_{e} \bar{L}_{L}\left(e_{R}^{H} H_{2}-e_{R} H_{1}\right) \\
& -y_{v} \bar{L}_{L}\left(v_{R} \tilde{H}_{1}+v_{R}^{H} \tilde{H}_{2}\right) \\
& -y_{e}^{\prime} \bar{e}_{L}^{H}\left(e_{R}^{H} \Phi_{2}-e_{R} \Phi_{1}\right) \\
& -y_{v}^{\prime} \bar{v}_{L}^{H}\left(v_{R} \Phi_{1}^{*}+v_{R}^{H} \Phi_{2}^{*}\right)+\text { H.c. }
\end{aligned}
$$

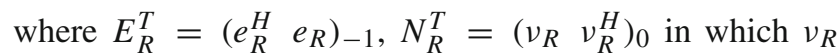
and $v_{R}^{H}$ are the right-handed neutrinos of the associated $S U(2)_{H}$ partner, while $e_{L}^{H}$ and $v_{L}^{H}$ are $S U(2)_{L, H}$ singlets with $Y\left(e_{L}^{H}\right)=-1$ and $Y\left(v_{L}^{H}\right)=0$, respectively. Note that the Yukawa couplings between the SM fermions and the neutral Higgses are flavor diagonal in G2HDM. On the other hand, the new Yukawa couplings are in general not flavor diagonal. To simplify the analysis, we fix the unitary mixing matrices among different flavors of heavy and SM fermions in the new Yukawa couplings with the $\mathcal{Z}_{2}$-odd scalars to be the identity matrix. As a result, the new fermions mass take 
Table 2 Classification of all the fields in G2HDM under $h$-parity

\begin{tabular}{ll}
\hline Fields & $h$-parity \\
\hline$h, G^{ \pm, 0}, \phi_{2}, G_{H}^{0}, \delta_{3}, f, W_{1,2,3}^{\mu}, B_{\mu}, X^{\mu}, W_{3}^{\mu \prime}, G^{\mu a}$ & 1 \\
$G_{H}^{p, m}, H_{2}^{0}, H_{2}^{0 *}, H^{ \pm}, \Delta_{p, m}, f^{H}, W_{1,2}^{\mu \prime}$ & -1 \\
\hline
\end{tabular}

a simple form

$m_{f^{H}}=\frac{1}{\sqrt{2}} y_{f^{H}} v_{\Phi}$.

Based on the signal search of the G2HDM gauge bosons, we set the minimum value of new fermion masses to be 1.0 $\mathrm{TeV}$ [34]

$m_{f^{H}}>1 \mathrm{TeV}$,

in contrast to the previous study where it was set to be greater than 1.5 TeV using the search of SUSY colored particle [52]. Moreover, we set the mass splitting between heavy fermions $f^{H}$ and dark matter $D$ to be

$m_{f^{H}}=1.05 m_{D}$

to study its effect in determining the DM relic abundance. This 5\% mass splitting, in contrast to the $20 \%$ adopted in [32], is more general than the previous study allowing the new fermions to contribute actively during the DM freezeout epoch.

\subsection{Dark matter candidate}

As we mentioned in the preceding sections, the stability of the DM in G2HDM is protected by accidental $\mathcal{Z}_{2}$ symmetry. This symmetry, also known as the hidden parity ( $h$-parity), is respected by all sector in G2HDM even after the SSB [31,32]. In addition, this parity also forbids FCNC [53,54] to occur at tree level for the SM fields in this model. All particles in G2HDM can be classified according to this parity as shown in Table 2.

In principle, all particles with zero electric charge and have $h$-parity equal to -1 are suitable DM candidates. Accordingly, from Table 2, the heavy neutrinos $v^{H}$, the neutral gauge boson $W^{\prime(p, m)}$, and the physical complex scalar $D$ satisfy these requirements. In this work, we focus on complex scalar $D$ as our DM candidate. The mass mixing matrix in Eq. (8) shows that the DM candidate, $D$, is linear combination of the interaction eigenstates $G_{H}^{p}, H_{2}^{0 *}$, and $\Delta_{p}$. Mathematically, this linear superposition is given by

$D=\mathcal{O}_{12}^{D} G_{H}^{p}+\mathcal{O}_{22}^{D} H_{2}^{0 *}+\mathcal{O}_{32}^{D} \Delta_{p}$, where $\mathcal{O}_{i j}^{D}$ is the $(i, j)$-th component of the rotation matrix $\mathcal{O}^{D}$. The values of these components depend on the numerical values of the parameters in Eq. (8).

Following Ref. [32], we classify our complex scalar DM candidate into three distinct cases: inert doublet-like DM, triplet-like DM, and Goldstone boson-like DM. This classification depends on the dominant component of the gauge eigenstate which can be characterized by the magnitude of $\mathcal{O}_{i j}^{D}$ in Eq. (16). We have inert doublet-like DM if $f_{H_{2}} \equiv\left(\mathcal{O}_{22}^{D}\right)^{2}>2 / 3$. Triplet-like DM is achieved if $f_{\Delta_{p}} \equiv\left(\mathcal{O}_{32}^{D}\right)^{2}>2 / 3$. Goldstone-like DM is characterized by $f_{G^{p}} \equiv\left(\mathcal{O}_{12}^{D}\right)^{2}>2 / 3$. The magnitude of the $\mathcal{O}_{i j}^{D}$ elements in Eq.(16) satisfy the following relation $f_{G}+f_{H_{2}}+f_{\Delta_{p}}=1$.

In addition, there is another condition that holds for the Goldstone-like DM. In this case, the value of $f_{G}$ must satisfy $0.67<f_{G^{p}}<0.8$ which is derived from both EWPT constraint and non-tachyonic DM mass solution as discussed in [32]. Here, we focus on triplet-like DM and Goldstonelike DM. The Inert doublet-like DM has been ruled out since it can not survive the relic density and direct detection constraints [32].

\section{Dark matter experimental constraints}

To understand the properties of DM, we evaluate the DMSM interactions via the existing experimental results. These include the observed DM relic abundance and the limit set by the null result coming from DM direct search. In this paper, we focus only on these two constraints as they provide the most stringent limit on the complex scalar DM phenomenology based on the previous study in [32]. Here, we discuss the general feature of these experimental limits used in this work.

\subsection{Relic density}

Since the interaction between DM and SM is very weak in most of the models, the observed relic abundance will be typically large. However, there exist some mechanisms other than DM self-annihilations which enable us to reach the observed abundance.

First, thanks to the common $\mathcal{Z}_{2}$-odd quantum number, DM coannihilates with other $\mathcal{Z}_{2}$-odd particles if their mass splitting is small (typically $\lesssim 10 \%$ ) such that their number densities do not suffer the Boltzmann suppression. In this work, coannihilations occur mainly between $D$ and new heavy fermions $f^{H}$. This happens because of $5 \%$ mass splitting that we impose on heavy fermions. Furthermore, as we will see later, this coannihilation is dominated by heavy fermions annihilation, especially heavy quarks-anti quarks 
pair $\left(q^{H} \bar{q}^{H}\right)$ annihilate into $q \bar{q}$ and $g g$. Here, $q \bar{q}$ and $g g$ denote SM quarks and gluon, respectively.

Second, the presence of SM Higgs and heavy Higgs $h_{2}$ resonances significantly increase the thermally-averaged DM annihilation cross section. In G2HDM, this mechanism allows us to reach the correct relic abundance.

We use the most recent result provided by the PLANCK collaboration [55] for the relic density, $\Omega h^{2}=0.120 \pm 0.001$, to restrict our thermal relic calculation. Moreover, we set an additional condition on the parameter space of G2HDM to reproduce this result within $2 \sigma$ significance.

\subsection{Direct detection}

The most up to date limit for DM direct search is provided by the XENON1T collaboration [56]. The zero signal result from this experiment strongly restricts the DM nucleon cross section, in particular, in the mass regime between $10 \mathrm{GeV}$ to $100 \mathrm{GeV}$. Furthermore, for DM mass around $25 \mathrm{GeV}$, they ruled out the DM-nucleon elastic cross sections which have the value larger than $10^{-46} \mathrm{~cm}^{2}$.

In G2HDM, it has been shown that the interaction between DM and nucleon exhibits isospin violation (ISV) [32]. In this case, the ratio between DM-neutron to DM-proton effective coupling, $f_{n} / f_{p}$, is not equal to 1 . This occurs due to the $Z_{i}$ bosons mediated interaction. As an example, for SM $Z$ exchange, the vectorial coupling between quark $q$ ( $d$ or $u$ type) and $Z$ is given by [32]

$$
\begin{aligned}
g_{\bar{q} q Z}^{V}= & \frac{i}{2}\left[\frac{g}{c_{W}}\left(T_{3}-2 Q_{q} s_{W}^{2}\right) \mathcal{O}_{11}^{G}\right. \\
& \left.+g_{H} T_{3}^{\prime} \mathcal{O}_{21}^{G}+g_{X} X \mathcal{O}_{31}^{G}\right] .
\end{aligned}
$$

Here, $Q_{q}, T_{3}, T_{3}^{\prime}$ and $X$ stand for the electric charge, the third generator of $S U(2)_{L}$, the third generator of $S U(2)_{H}$, and the generator of $U(1)_{X}$, respectively. Since the $u$ and $d$ quark have distinct quantum number assignments with respect to the underlying gauge group, they couple to the SM $Z$ differently.

In order to accommodate the ISV, we need to calculate the DM-nucleus elastic scattering cross section $\sigma_{D \mathcal{N}}$

$\sigma_{D \mathcal{N}}=\frac{4 \mu_{\mathcal{A}}^{2}}{\pi}\left[f_{p} \mathcal{Z}+f_{n}(\mathcal{A}-\mathcal{Z})\right]^{2}$,

where $\mathcal{N}$ denotes a nucleus with mass number $\mathcal{A}$ and proton number $\mathcal{Z}$ and $\mu_{\mathcal{A}}=m_{D} m_{\mathcal{A}} /\left(m_{D}+m_{\mathcal{A}}\right)$ is the reduced mass for DM-nucleus system. For definiteness, we neglect all the isotopes of xenon and fix $\mathcal{A}$ and $\mathcal{Z}$ to 131 and 54, respectively. To extract the DM-nucleon effective couplings $f_{n}$ and $f_{p}$, we employ micrOMEGAs [57] in our computation. To compare against the limit given by XENON1T which assumes $f_{n}=f_{p}$ in their analysis, we need to reconstruct their result at the nucleus level. In order to do so, we follow the same procedure in [32] and obtain the following expression for general value of $f_{n} / f_{p}$

$\sigma_{D \mathcal{N}}^{\mathrm{X} 1 \mathrm{~T}}=\sigma_{p}^{\mathrm{SI}}(\mathrm{X} 1 \mathrm{~T}) \times \frac{\mu_{\mathcal{A}}^{2}}{\mu_{p}^{2}} \times\left[\mathcal{Z}+\frac{f_{n}}{f_{p}}(\mathcal{A}-\mathcal{Z})\right]^{2}$,

where $\mu_{p}^{2}$ is the DM-proton reduced mass. In this paper, we use Eq. (19) to constrain our direct detection prediction.

In general, the DM-nucleon interaction will be different from antiDM-nucleon interaction. The spin independent interaction for complex scalar DM can be obtained by using the effective operator language as [58]

$$
\begin{aligned}
\mathcal{L}_{D}= & 2 \lambda_{N, e} M_{D} D D^{*} \bar{\psi}_{N} \psi_{N}+i \lambda_{N, o} \\
& \times\left(D^{*} \overleftrightarrow{\partial_{\mu}} D\right) \bar{\psi}_{N} \gamma^{\mu} \psi_{N},
\end{aligned}
$$

where the $\psi_{N}, \lambda_{N, e}$, and $\lambda_{N, o}$ stand for the nucleon field operator, the coupling of even operator, and the coupling of odd operator, respectively. In this case, the effective coupling between DM (antiDM) and the nucleon is

$\lambda_{N}=\frac{\lambda_{N, e} \pm \lambda_{N, o}}{2}$,

where the plus (minus) sign denotes DM-nucleon (antiDMnucleon) interaction. Under the interchange between $D$ and $D^{*}$, the first (second) term on the right hand side of Eq. (20) stays the same (flips sign). Because of this, the first term is called even operator while the second term describes odd operator. As a consequence, the strength of the interaction between DM-nucleon and antiDM-nucleon is different and it is given by Eq. (21). Hence, the value of $\sigma_{D^{*} \mathcal{N}}$ is in general not equal to $\sigma_{D \mathcal{N}}$ given by Eq. (18) because the effective couplings $f_{p}$ and $f_{n}$ for $D$ are different from those for $D^{*}$. In this work, we average the contribution from both $\sigma_{D \mathcal{N}}$ and $\sigma_{D^{*} \mathcal{N}}$ when comparing our calculation with the XENON1T result given in Eq. (19).

The Feynman diagrams relevant for describing DM-quark interactions in G2HDM are shown in Fig. 1. For the upper part, the left panel is the $t$-channel interaction mediated by three Higgs bosons while the right panel is the $t$-channel with three neutral gauge bosons exchange. In addition, heavy quarks exchange via s and t-channel are shown in the lower part of the same figure. There is a destructive interference between heavy quarks mediated diagrams and the Higgs mediated diagrams in triplet-like DM $\left(\Delta_{p}\right)$. On the other hand, the diagrams with the neutral gauge bosons and heavy quarks mediators add up constructively in Goldstone bosonlike $\mathrm{DM}\left(G_{H}^{p}\right)$. 
Fig. 1 The Feynman diagrams relevant for DM direct search. They consist of three t-channel diagrams with the $\mathcal{Z}_{2}$-even Higgs bosons (top left), neutral gauge bosons (top right) and heavy quarks (bottom left) mediators. There is also s-channel heavy quarks exchange (bottom right)
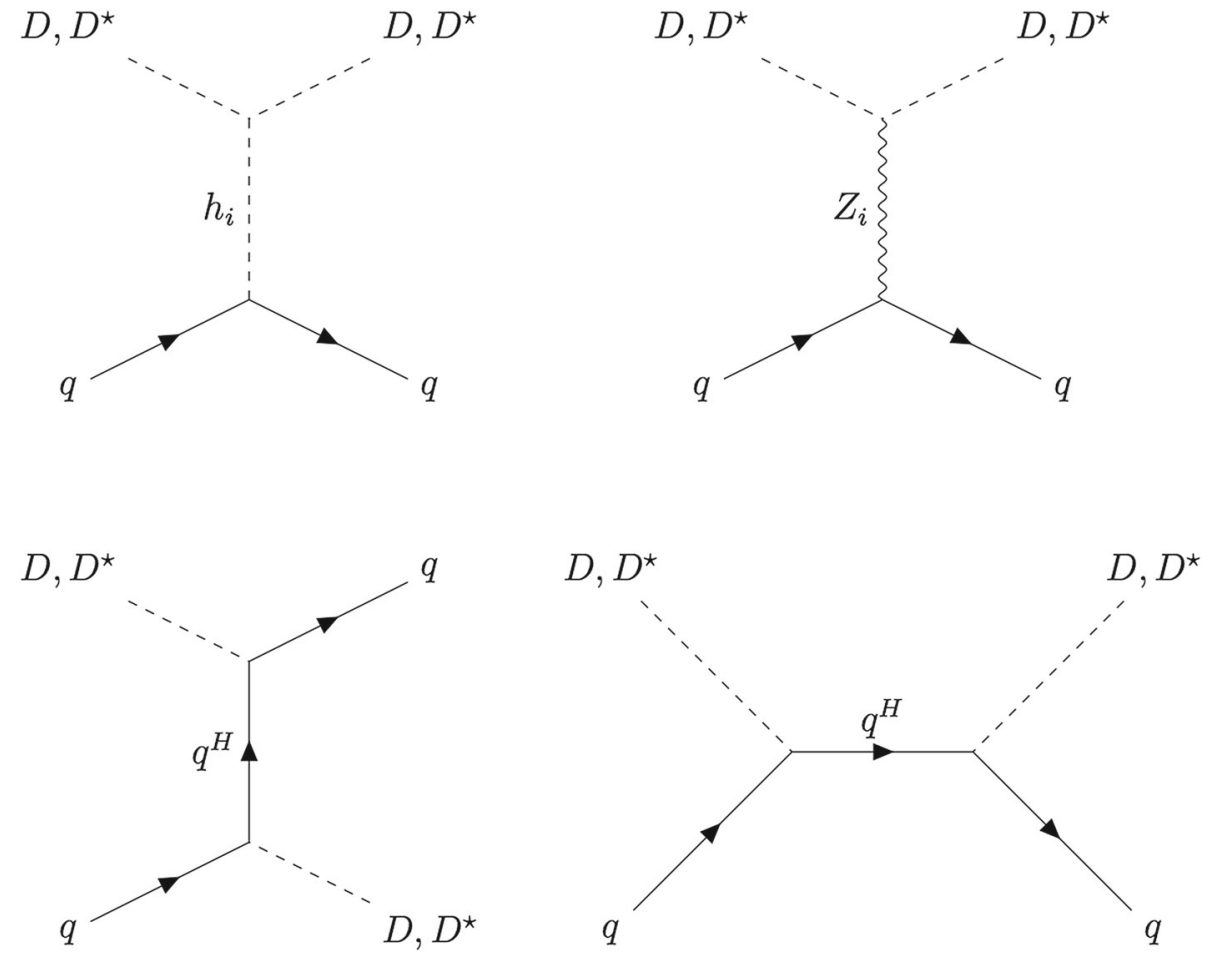

\section{Numerical analysis and results}

\subsection{Methodology}

Taking into account the various constraints based on previous G2HDM studies, we collect a sample of points by doing random scan. These points pass the scalar sector constraints studied in [36] as we mentioned in the preceding section. In addition, we also impose the limits from the gauge sector carried out in [37]. This study includes Drell-Yan constraint which restricts the $S U(2)_{H}$ gauge coupling, $g_{H}$, below 0.1 . To prevent the decay of DM into $W^{\prime}$, we impose the condition $m_{W^{\prime}}>m_{D}$ which translates into the lower bound of $g_{H}$ [32]

$g_{H \min }=\frac{2 m_{D}}{\sqrt{v^{2}+v_{\Phi}^{2}+4 v_{\Delta}^{2}}}$.

The search of $Z^{\prime}$ enforces us to scan $v_{\Phi}$ in the mass range between $20 \mathrm{TeV}$ to $100 \mathrm{TeV}$ [37]. Moreover, we fix $M_{X}=$ $2 \mathrm{TeV}$ to satisfy heavy $M_{X}$ scenario while maintaining the SM-like $Z$ to $91.1876 \pm 0.0021 \mathrm{GeV}$ within $3 \sigma$ significance [37].

As discussed in previous section, we set lower limit of heavy fermion masses to be $1 \mathrm{TeV}$ together with $5 \%$ mass splitting between heavy fermions and the dark matter. Furthermore, we take the new Yukawa couplings, which are related to the new heavy fermion masses by $m_{f^{H}}=$ $y_{f H} v_{\Phi} / \sqrt{2}$, to be small enough in order to suppress their contributions on perturbative unitarity and renormalization group running effects. To accommodate all of these require-
Table 3 Parameter ranges used in the numerical scans that pass SGSC constraints. Here, $M_{X}$ is fixed at $2 \mathrm{TeV}$ and $M_{Y}=0$

\begin{tabular}{lll}
\hline Parameter & Triplet-like & Goldstone-like \\
\hline$\lambda_{H}$ & {$[0.12,2.75]$} & {$[0.12,2.75]$} \\
$\lambda_{\Phi}$ & {$\left[10^{-4}, 4.25\right]$} & {$\left[10^{-4}, 4.25\right]$} \\
$\lambda_{\Delta}$ & {$\left[10^{-4}, 5.2\right]$} & {$\left[10^{-4}, 5.2\right]$} \\
$\lambda_{H \Phi}$ & {$[-6.2,4.3]$} & {$[-6.2,4.3]$} \\
$\lambda_{H \Delta}$ & {$[-4.0,10.5]$} & {$[-4.0,10.5]$} \\
$\lambda_{\Phi \Delta}$ & {$[-5.5,15.0]$} & {$[-5.5,15.0]$} \\
$\lambda_{H \Phi}^{\prime}$ & {$[-1.0,18.0]$} & {$[-1.0,18.0]$} \\
$\lambda_{H}^{\prime}$ & {$[-8 \sqrt{2} \pi, 8 \sqrt{2} \pi]$} & {$[-8 \sqrt{2} \pi, 8 \sqrt{2} \pi]$} \\
$g_{H}$ & {$[$ See text, 0.1$]$} & {$[S e e t e x t, 0.1]$} \\
$g_{X}$ & {$\left[10^{-8}, 1.0\right]$} & {$\left[10^{-8}, 1.0\right]$} \\
$M_{H \Delta} / \mathrm{GeV}$ & {$[-3000,5000]$} & {$[0.0,5000]$} \\
$M_{\Phi \Delta} / \mathrm{GeV}$ & {$[-50.0,50.0]$} & {$[0.0,700]$} \\
$v_{\Delta} / \mathrm{TeV}$ & {$[0.5,20.0]$} & {$[14.0,20.0]$} \\
$v_{\Phi} / \mathrm{TeV}$ & {$[20,100]$} & {$[20,28.0]$} \\
\hline
\end{tabular}

ments, we adopt the Yukawa couplings for each point in our random scan as follows

$y_{f^{H}}=\max \left[\frac{1.0 \mathrm{TeV}}{v_{\Phi} / \sqrt{2}}, \min \left(\frac{1.05 m_{D}}{v_{\Phi} / \sqrt{2}}, 1\right)\right]$.

From this formula, it is easy to see that the value of $y_{f^{H}}$ is always less than 1 for all parameter space.

Approximately, 3 million points are collected from our random scan. These points cover all numerical values for model parameters, gauge bosons and scalar masses, as well 
as all components of three mixing matrices $\mathcal{O}, \mathcal{O}^{D}$ and $\mathcal{O}^{G}$. Furthermore, we input these numbers to MicrOMEGAs [57] to compute DM relic density and DM-nucleon cross section.

The scan range for our computation is tabulated in Table 3 . Note that $M_{H \Delta}, M_{\Phi \Delta}, v_{\Delta}$ and $v_{\Phi}$ have distinctive ranges for the associated DM composition. These differences are remarkable in the case of $v_{\Delta}$ and $v_{\Phi}$ in the Goldstone-like column which exhibits a fine-tuning. This happens because the Goldstone-like composition is achievable only when $0.8 \leq v_{\Delta} / v_{\Phi}<0.9$ as discussed in [32].

\subsection{Results}

Here, we discuss the analysis of our numerical results. We evaluate the effects of heavy fermions on DM phenomenology for triplet-like DM and Goldstone-like DM separately.

\section{$S U(2)_{H}$ triplet-like DM}

The scatter plots for the relic density versus DM mass $m_{D}$ in the triplet-like DM are presented in Fig. 2. The red line represents the observed relic density provided by the PLANCK collaboration. The right panel takes heavy fermions contributions into account while the left is adopted from [32]. Let us elaborate more by considering different DM mass regimes.

(i) We start with the lower DM mass regime ranging from $1 \mathrm{GeV}$ to $62.5 \mathrm{GeV}$. In this region, the $\mathrm{DM}$ annihilation cross section is dominated by the following processes: $D D^{*} \rightarrow c \bar{c}, \tau^{+} \tau^{-}$and $b \bar{b}$, occurring mainly via the $s$-channel of the Higgs bosons exchange. This can be understood based on the $D D^{*} h_{i}$ coupling in Eq. (A2). Thanks to the large value of $v_{\Phi}$, the $h_{2}\left(\delta_{3}\right.$-like) mediated diagram is equally as important as the $h_{1}$ (SM-like) exchange while the heaviest Higgs $h_{3}$ ( $\phi_{2}$-like) remains subdominant [32]. The observed relic abundance can be achieved in this wide range $\left(12 \mathrm{GeV}<m_{D} \leq 62 \mathrm{GeV}\right)$ due to the various combinations for the $D D^{*} h_{i}$ coupling in Eq. (A2). However, this picture is only valid when heavy fermions are neglected (the left panel of Fig. 2). Once they are included (the right panel of Fig. 2), the $\mathrm{t}$-channel of heavy fermions exchange becomes relevant. There is a cancellation between the Higgs and new heavy fermions mediated diagrams which reduces the value of DM annihilation cross section. This enhances the relic density as shown in the right panel of Fig. 2 where the gray points are shifted above the horizontal red line. The observed relic density is achieved in the mass range between $44 \mathrm{GeV}$ to $62 \mathrm{GeV}$.

(ii) When DM mass approaches half of the SM Higgs mass $\left(m_{D} \approx 62.5 \mathrm{GeV}\right)$, the interactions involving the s- channel of $h_{1}$ exchange become dominant. This enhances the annihilation cross section resulting in the suppression of the relic density. However, due to the wide variation of the $D D^{*} h_{1}$ coupling in Eq. (A2), the observed relic abundance can still be achieved. This is also true for $h_{2}$ resonance. In this case, the resonance appears in a broad range of DM mass thanks to the arbitrary values of $m_{h_{2}}$ $\left(m_{h_{2}}>m_{h_{1}}\right)$. Note that there is no SM $Z$ resonance because its coupling is suppressed by $\left(\mathcal{O}_{32}^{D}\right)^{2} \mathcal{O}_{21}^{G}$ as can be seen from Eq. (A1).

(iii) Above the SM Higgs resonance, $m_{h_{1}} / 2<m_{D}<$ $500 \mathrm{GeV}$, the major contributions to the DM annihilation cross section are given by $W^{+} W^{-}$(more than $\sim 50 \%$ ), $h_{1} h_{1}(\sim 25 \%)$, and $Z Z(\sim 20 \%)$ final states [32]. In all of these final states, the main contributions come from the $h_{1}$ and $h_{2}$ exchanges which lead to the $S$-wave cross section. On the other hand, the $Z_{i}$ mediated diagrams relevant for $W^{+} W^{-}$final states are $P$-wave suppressed.

(iv) Next, for heavy DM mass regime $\left(m_{D}>500 \mathrm{GeV}\right)$, the dominant final states are given by the longitudinal parts of the SM gauge bosons, $W_{L}^{+} W_{L}^{-}$and $Z_{L} Z_{L}$. However, this is only valid if one neglects heavy fermions contribution. This picture changes in the mass regime larger than $1 \mathrm{TeV}$ in the presence of heavy fermions.

(v) Finally, in the $\mathrm{TeV}$ region $\left(m_{D}>1 \mathrm{TeV}\right)$, heavy fermions coannihilations start to dominate. This can be seen from the right panel of Fig. 2. The main coannihilation channels in this regime are given by heavy quarks annihilations into a pair of SM quark-anti quark and gluon: $q^{H} \bar{q}^{H} \rightarrow q \bar{q}, g g$. Based on the points that we collect from our scan, these processes contribute around $87 \%$ in this regime. This is expected since these are the QCD interactions controlled by the strong coupling $\alpha_{S}$. The main diagrams associated with these interactions are given by the s-channel of gluon exchange for $q \bar{q}$ final states and the s-channel of gluon exchange, $t$ - and u-channel of $q^{H}$ exchange for $g g$ final states as shown in Fig. 3.

In Ref. [32], the relevant diagrams for the spin independent DM-nucleon interactions are given by the t-channel of $h_{i}$ and $Z_{i}$ exchange as displayed in the upper part of Fig. 1. The leading contribution comes from $h_{1}$ mediator while $h_{2}, Z$, and $Z^{\prime}$ mediators give non-negligible effects. This is summarized in the left panel of Fig. 4, adopted from [32]. The XENON1T limit is described by the solid red curve which assumes $f_{n} / f_{p}=1$. The orange points in this figure are allowed by the relic density and direct detection constraints. As one can see, for DM mass larger than $300 \mathrm{GeV}$, both constraints can be evaded. As a result of ISV originating from $Z$ and $Z^{\prime}$ exchange, there are some orange points which are located slightly above the XENON1T exclusion limit. 
Fig. 2 The left panel describes triplet-like DM SGSC surviving regions projected on $\left(m_{D}\right.$, $\Omega_{D} h^{2}$ ) adopted from [32]. This figure neglects heavy fermions contributions. The inclusion of heavy fermions (with 5\% mass splitting) is depicted on the right panel. For both figures, there are no resonances and coannihilations in the gray area

Fig. 3 Dominant Feynman diagrams relevant for coannihilation in $\mathrm{TeV}$ regime
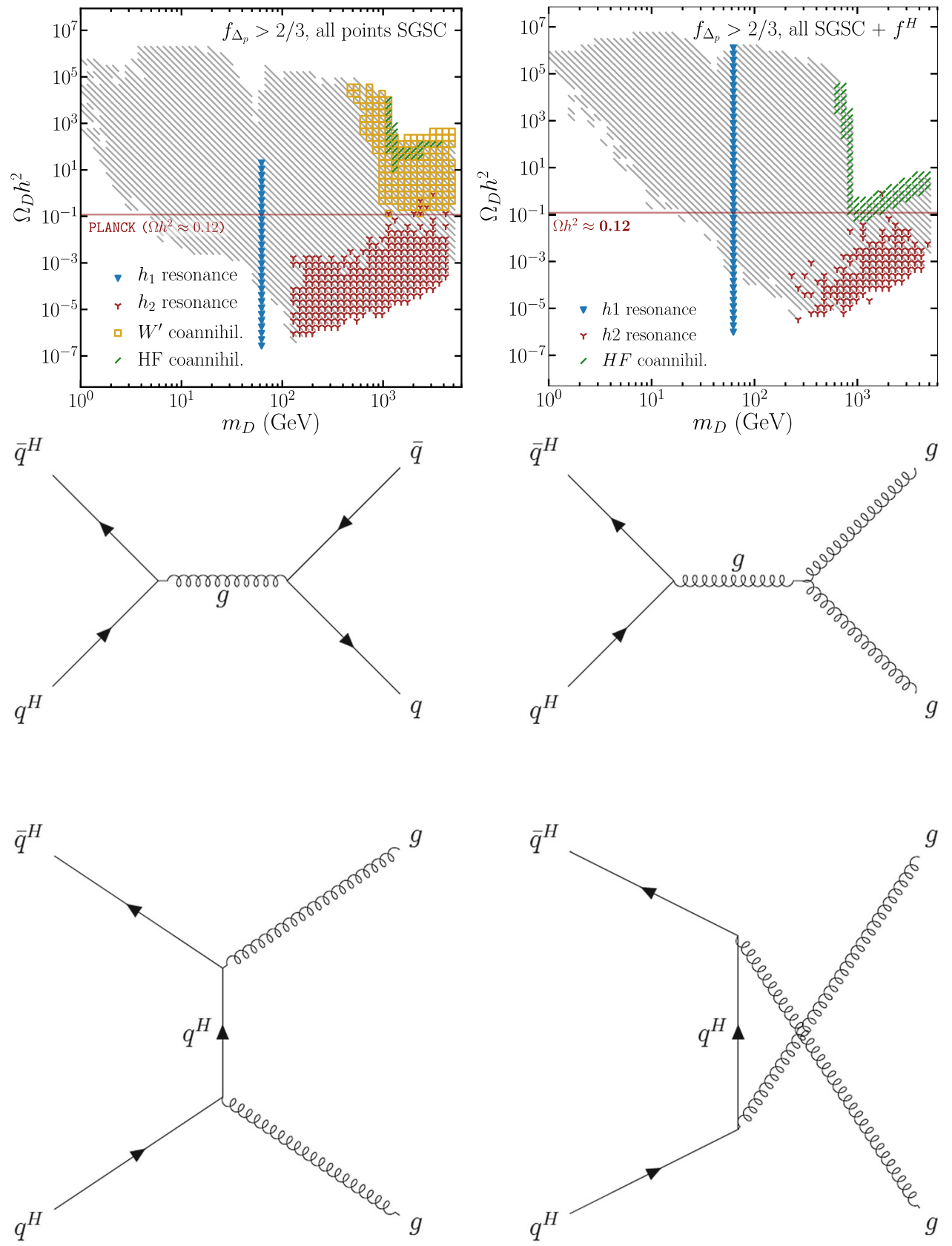

As a comparison, the corresponding XENON1T limit for $f_{n} / f_{p}=-0.5$ is also shown.

On the other hand, when heavy fermions contribution is considered, one needs to include all diagrams in Fig. 1. There is a destructive interference between heavy quarks and the Higgs mediated diagrams. This cancellation can be extracted from the sum of these diagrams as

$\mathcal{A}_{\left(q^{H}+h_{i}\right)} \propto\left[\frac{2 m_{q^{H}}\left(\mathcal{O}_{12}^{D}\right)^{2}}{v_{\Phi}^{2}}+\frac{m_{q} \mathcal{O}_{1 i} \lambda_{D D^{*} h_{i}}}{v m_{h_{i}}^{2}}\right]$,

where $m_{q}$ stands for the mass of the SM quarks. The index $i$ runs from 1 to 2 denoting the contributions from the Higgs $h_{1}$ and $h_{2}$ while the coupling $\lambda_{D D^{*} h_{i}}$ is given by Eq. (A2). The first term on the right hand side of Eq. (24) which corresponds to heavy fermions exchange is always positive while the second two terms associated to the Higgses exchange, for both $h_{1}$ and $h_{2}$, are mostly negative. In addition, the cancellation given by Eq. (24) is also relevant in the region (i) of the thermal relic calculation as discussed above with a factor of $1 / 2$ in front of the first term. This factor stems from the fact that only t-channel of heavy fermions exchange contributes to the DM annihilation cross section.

The effect of cancellation given by Eq. (24) can be seen from the right panel of Fig. 4 where most of the gray points are located below $10^{-42} \mathrm{~cm}^{2}$. Interestingly, this cancellation 
Fig. 4 In the left panel, adopted from [32] (without heavy fermions), the red solid curve is the official XENON1T exclusion limit without ISV, while the green solid curve describes the same limit with ISV value $f_{n} / f_{p}=-0.5$. Heavy fermions contribution is shown in the right panel. Here, the green solid line is XENON1T limit with $f_{n} / f_{p}=-0.6$. For both figures, the orange filled squares pass both RD and DD constraints
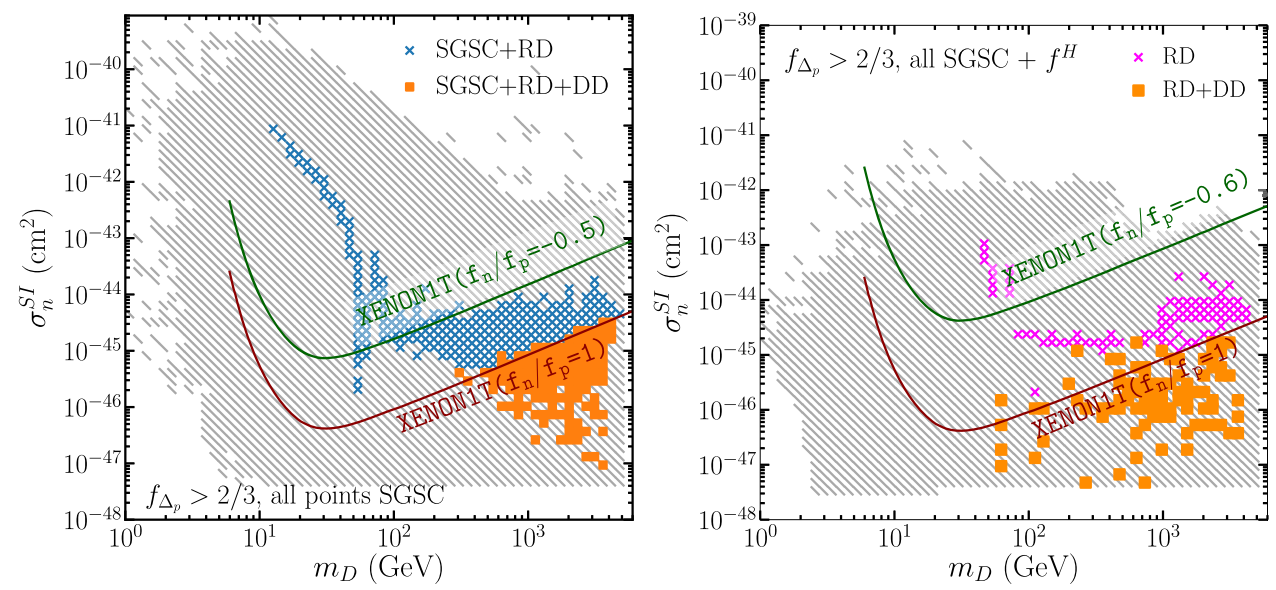

shifts the allowed DM mass (orange squares area) towards the lighter region, reaching up to $54 \mathrm{GeV}$. There are several gaps between $64 \mathrm{GeV}$ and $100 \mathrm{GeV}$ as well as $140 \mathrm{GeV}$ and $200 \mathrm{GeV}$ where none of the points survive both relic density and direct detection constraints. This is because the cancellation given by Eq. (24) does not work effectively in this regime. In addition, the existence of the orange points significantly above the XENON1T limit shows the importance of the $Z$ and $Z^{\prime}$ exchange when this cancellation happens efficiently.

We see that for triplet-like DM, the inclusion of heavy fermions put a stronger constraint on the observed relic abundance, especially in the $\mathrm{TeV}$ regime. In contrast, they reduce the DM-nucleon spin independent cross section alleviating the current limit from XENON1T experiment.

\section{$S U(2)_{H}$ Goldstone boson-like DM}

As we mentioned before, the Goldstone-like DM is dominated by $G_{H}^{p}$. However, in this particular case, the allowed composition lies in a very narrow regime between $0.67<$ $f_{G}<0.8$. As a result, this DM lives in a tiny region in the parameter space of G2HDM model. In the left panel of Fig. 5, we show the scatter plot of the DM relic abundance with respect to its mass. The underlying physics in each mass regime (regions (i)-(iv)) is mostly similar to the triplet case [32]. Moreover, when heavy fermions are included, as shown in the right panel of Fig. 5, they strongly dominate the coannihilation channels in $\mathrm{TeV}$ regime as in triplet-like DM case (region (v)). Furthermore, there is no cancellation between Higgs and heavy fermions exchange thanks to the limited parameter space.

The scatter plot for DM-neutron cross section versus DM mass in the absence of heavy fermions is presented in the left panel of Fig. 6 . The leading diagram is given by the t-channel of $h_{1}$ exchange which is significant in the mass range between $3 \mathrm{GeV}$ and $100 \mathrm{GeV}$. The next important diagrams come from $Z^{\prime}$ and $Z$ exchange [32]. These ISV diagrams are relevant for DM mass larger than $100 \mathrm{GeV}$.
As a result of the interference between these three dominant diagrams, the spin independent cross section varies in a wide range. However, only the orange points in the mass range $200 \mathrm{GeV} \leq m_{D} \leq 600 \mathrm{GeV}$ satisfy both constraints from the observed relic density and the published XENON1T limit. These points have $f_{n} / f_{p}=-1.86$ thanks to the fine-tuned parameter space. As shown in the right panel of Fig. 6, the inclusion of heavy fermions does not significantly change this result. They add up constructively with the $Z^{\prime}$ and $Z$ mediated diagrams shifting the ISV value of the orange region to have $f_{n} / f_{p}=-2.0$. This enhancement is clearly seen in the $\mathrm{TeV}$ regime where the gray points are shifted upward.

\section{QCD Sommerfeld correction and bound state formation}

Based on the previous section, we see that the contributions of heavy fermions are quite significant, especially in the $\mathrm{TeV}$ regime of the DM mass. In this case, for both triplet-like and Goldstone-like DM, heavy quarks annihilations $\left(q^{H} \bar{q}^{H} \rightarrow q \bar{q}, g g\right)$ strongly reduce the relic abundance due to the QCD interactions. Since these processes occur in the early universe, at energy scale much higher than the quark-hadron phase transition era, heavy quarks experience a long range interaction via gluon exchange. This interaction can be described by Coulomb-like potential as [59]

$V(r)=-\frac{\zeta^{\prime}}{r}, \quad \zeta^{\prime}=\left(C_{q^{H}}-\frac{3}{2}\right) \alpha_{s}$,

where $\zeta^{\prime}$ describes the modified coupling between $q^{H}$ and $\bar{q}^{H}$ free pair which depends on the quadratic Casimir coefficient of the color representation of the heavy quark $C_{q H}$. The presence of the Coulomb-like potential modifies the perturbative calculation. This effect is known as the Sommerfeld correction [60]. For heavy quarks, the value of $C_{q^{H}}$ is equal to $4 / 3$ since they belong to the color triplet representation 
Fig. 5 The left panel shows Goldstone-like DM SGSC allowed regions projected on $\left(m_{D}, \Omega_{D} h^{2}\right)$. This figure, adopted from Ref. [32], neglects heavy fermions contributions. In contrast, the figure in the right panel takes heavy fermions into account which reduce the relic density in the $\mathrm{TeV}$ regime

Fig. 6 The left panel is taken from [32] which omits heavy fermions. They are included in the figure on the right panel. The red solid curve, in both figures, is the XENON1T limit without ISV. The green solid line in the left (right) describes the XENON1T exclusion line with ISV value $f_{n} / f_{p}=-1.5$ $\left(f_{n} / f_{p}=-0.55\right)$. The small region of orange squares shows the fine-tuned region with $f_{n} / f_{p}=-1.86$ for the left panel and $f_{n} / f_{p}=-2.00$ for the right panel. These areas are allowed by RD and DD constraints
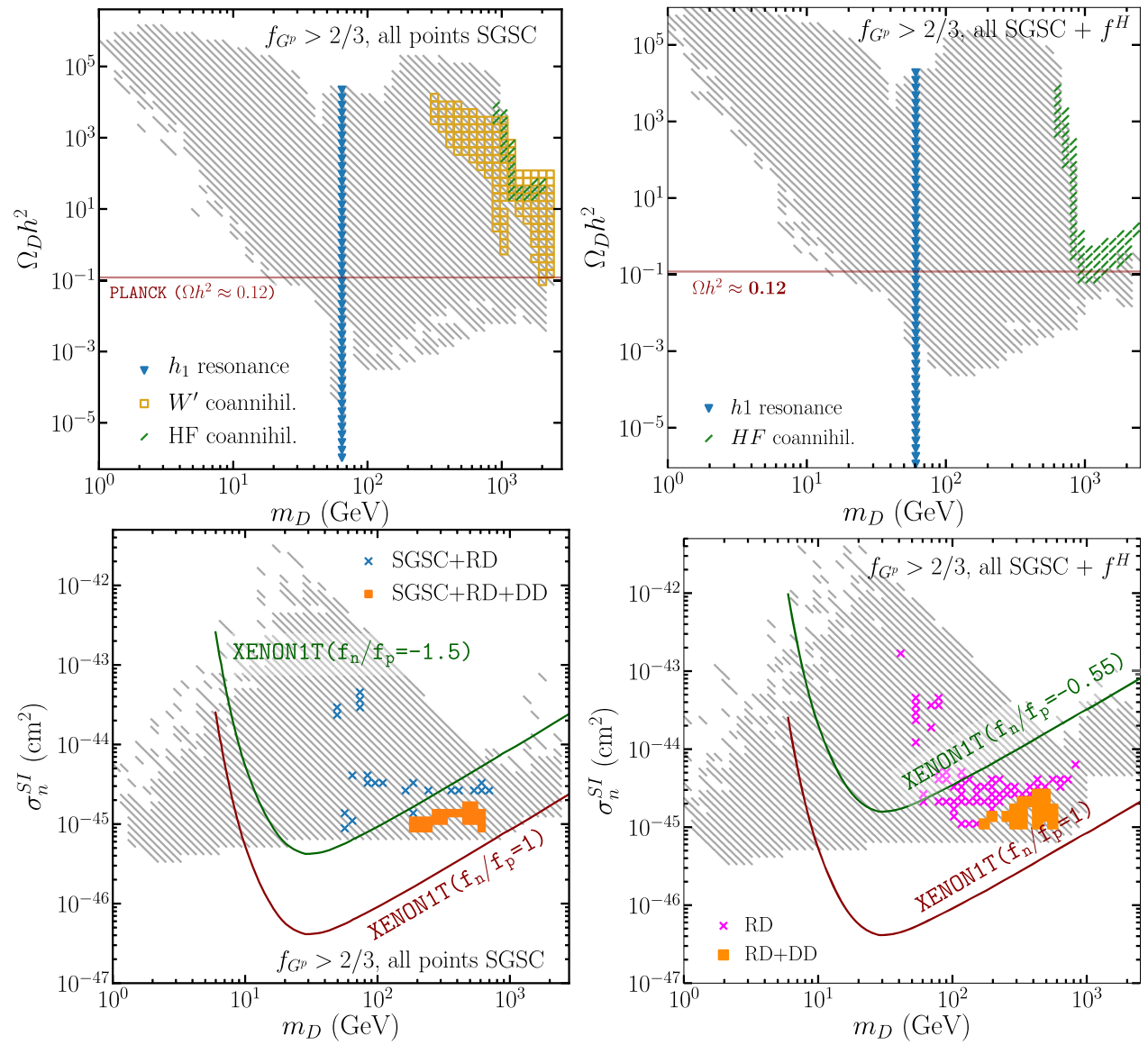

of $S U(3)_{C}$. Furthermore, the presence of this potential may induce the bound state formation of $q^{H} \bar{q}^{H}$ pairs in the early universe (see [61-85] for detail study of the bound state effect on DM relic calculation). Therefore, one needs to consider these two effects when calculating the DM abundance. In this work, we follow the method introduced in [85] for our QCD Sommerfeld correction and QCD bound state effect calculation.

\subsection{QCD Sommerfeld correction}

The thermally averaged $S$ - and $P$-wave annihilation cross sections of $q^{H} \bar{q}^{H}$ into pair of SM quark and gluons can be expanded as

$\left\langle\sigma v_{r e l}\right\rangle=a+b\left(T / m_{q^{H}}\right)+\mathcal{O}\left(\left(T / m_{q^{H}}\right)^{2}\right)$,

where $a$ and $b$ denote the $S$ - and $P$-wave term, respectively. For $q \bar{q}$ final state, the corresponding expression is given by [85-87]

$\left\langle\sigma v_{r e l}\right\rangle_{q \bar{q}}=\frac{\pi \alpha_{s}^{2}}{m_{q^{H}}^{2}}\left[\frac{4}{3}-\frac{14}{3}\left(T / m_{q^{H}}\right)\right]$, while for gluon pair final state, the associated cross section is

$\left\langle\sigma v_{r e l}\right\rangle_{g g}=\frac{\pi \alpha_{s}^{2}}{m_{q^{H}}^{2}}\left[\frac{7}{27}+\frac{1}{6}\left(T / m_{q^{H}}\right)\right]$.

In general, the presence of the Coulomb potential of the form $V(r)=-\alpha / r$ will affect both $S$ - and $P$-wave terms. For the $S$-wave term, it becomes $a S\left(\alpha / v_{r e l}\right)$ where

$S\left(\alpha / v_{r e l}\right)=\frac{2 \pi \alpha / v_{r e l}}{1-e^{-2 \pi \alpha / v_{r e l}}}$,

where $v_{r e l}$ stands for the relative velocity between $q^{H}$ and $\bar{q}^{H}$. The Sommerfeld correction enhances the annihilation cross section if the potential is attractive $(\alpha>0)$ while the suppression occurs when the potential is repulsive $(\alpha<0)$. Taking this correction into account in the early universe, we need to evaluate the thermally-averaged Sommerfeld $S$-wave cross section as

$a\left\langle S\left(\alpha / v_{r e l}\right)\right\rangle=a \int_{0}^{\infty} S\left(\alpha / v_{r e l}\right) f\left(v_{r e l}\right) d v_{r e l}$, 
where the velocity distribution $f\left(v_{r e l}\right)$ is given by the Maxwell-Boltzmann distribution

$f\left(v_{r e l}\right)=\left(\frac{\mu}{2 \pi T}\right)^{3 / 2} 4 \pi v_{r e l}^{2} e^{-\frac{\mu v_{r e l}^{2}}{2 T}}$,

where $\mu=m_{q^{H}} / 2$ and $T$ are the reduced mass of the $q^{H} \bar{q}^{H}$ system and the temperature, respectively. In this work, we only consider the Sommerfeld correction in the $S$-wave term.

Following two body color decomposition for the $S$-wave cross section, the thermally-averaged Sommerfeld corrections are [83]

$$
\begin{aligned}
& \left\langle\sigma v_{r e l}\left(q^{H} \bar{q}^{H} \rightarrow g g\right)\right\rangle_{s, \text { Somm }} \\
& =\left\langle\sigma v_{r e l}\right\rangle_{s, g g}\left(\frac{2}{7}\left\langle S\left(\frac{4 \alpha_{s} / 3}{v_{r e l}}\right)\right\rangle+\frac{5}{7}\left\langle S\left(\frac{-\alpha_{s} / 6}{v_{r e l}}\right)\right\rangle\right), \\
& \left\langle\sigma v_{r e l}\left(q^{H} \bar{q}^{H} \rightarrow q \bar{q}\right)\right\rangle_{s, \text { Somm }} \\
& =\left\langle\sigma v_{r e l}\right\rangle_{s, q \bar{q}}\left\langle S\left(\frac{-\alpha_{s} / 6}{v_{r e l}}\right)\right\rangle,
\end{aligned}
$$

where $\left\langle\sigma v_{r e l}\right\rangle_{s, q \bar{q}}$ and $\left\langle\sigma v_{r e l}\right\rangle_{s, g g}$ denote the $S$-wave part of Eqs. (27) and (28), respectively.

\subsection{QCD bound state formation}

When $q^{H}$ and $\bar{q}^{H}$ form a bound state $\eta$, the Coulomb-like potential due to gluon exchange becomes

$$
\begin{aligned}
V(r) & =-\frac{\zeta}{r}, \\
\zeta & =\frac{1}{2}\left(C_{q^{H}}+C_{\bar{q}^{H}}-C_{\eta}\right) \alpha_{s},
\end{aligned}
$$

where $C_{\eta}$ is the quadratic Casimir operator of the $q^{H} \bar{q}^{H}$ bound state in a particular color state. Here, for color singlet bound state $C_{\eta}=C_{\mathbf{1}}=0$. Furthermore, we only consider bound states with total angular momentum $L=0$ and spin $S=0$. The corresponding binding energy of the bound state $E_{B}$ and its Bohr radius $a$ are

$$
\begin{aligned}
E_{B} & =\frac{\zeta^{2} \mu}{2}, \\
a & =(\zeta \mu)^{-1},
\end{aligned}
$$

where $\mu=m_{q^{H}} / 2$ is the reduced mass of the $q^{H}$ and $\bar{q}^{H}$ system.

To calculate the bound state formation cross section, one needs to know the integrated dissociation cross section first. A $q^{H}$ and $\bar{q}^{H}$ bound state can be destroyed by absorbing a gluon. The dissociation cross section averaged over the incoming gluon color is [81]

$\sigma_{d i s}=\frac{1}{8} \times \frac{4}{3} \times \frac{2^{9} \pi^{2}}{3} \alpha_{s} a^{2}\left(\frac{E_{B}}{\omega}\right)^{4} \frac{1+v^{2}}{1+(\kappa \nu)^{2}}$

$$
\times \frac{e^{-4 v \operatorname{arccot}(\kappa \nu)-2 \pi v}}{1-e^{-2 \pi \nu}} \kappa^{-1} .
$$

In this expression we define $\nu, \kappa$, and $\omega$ as

$v \equiv \frac{\left|\zeta^{\prime}\right|}{v_{r e l}}, \quad \kappa \equiv \frac{\zeta}{\left|\zeta^{\prime}\right|}, \quad \omega \approx E_{B}+\frac{1}{2} \mu v_{r e l}^{2}$.

Equation (37) describes the dissociation cross section of a bound state after absorbing a gluon with energy $\omega$. The first factor in this equation is the averaged over incoming gluon color while the second number denotes the color factor of the bound state wave function. The bound state formation cross section can be obtained by using the Milne relation as

$\sigma_{b s f}=\frac{g_{\eta} g_{g} \omega^{2}}{g_{q^{H}} g_{\bar{q}^{H}}\left(\mu v_{r e l}\right)^{2}} \sigma_{d i s}$,

where $g_{g}=16, g_{q^{H}}=g_{\bar{q}^{H}}=12$, and $g_{\eta}=1$ are the degree of freedom of gluon, $q^{H}, \bar{q}^{H}$, and color singlet bound state, respectively.

In addition, a $q^{H}$ and $\bar{q}^{H}$ bound state experiences annihilation decay and individual decay of each $q^{H}\left(\bar{q}^{H}\right)$. In the former case, the $q^{H}$ and $\bar{q}^{H}$ annihilate into the SM particles while in the latter case, each $q^{H}\left(\bar{q}^{H}\right)$ decays into DM and SM particle. Thanks to the 5\% mass splitting between the $\mathrm{DM}$ and heavy quarks, the latter process is suppressed. Additionally, this decay channel is further suppressed by $\left(\mathcal{O}_{12}^{D}\right)^{2}$. Therefore, in our case, we only need to consider the annihilation decay channel. The main decay mode is given by two-gluon final state [88]

$\Gamma_{\eta}^{q^{H}}=\frac{2}{3} \mu \alpha_{s}^{2} \zeta^{3}$

Note that in Eq. (40), the explicit strong coupling $\alpha_{s}$ is evaluated at the scale of $2 m_{q^{H}}$ while the inverse Bohr radius $a^{-1}$ is used when evaluating the $\alpha_{s}$ dependence in $\zeta$.

Considering the DM relic, we have to take the thermal average of the bound state effect. To get rid of the temperature dependence when evaluating the thermal average, we define $z \equiv E_{B} / T$ and $u \equiv \frac{1}{2} \mu v_{r e l}^{2} / T$ such that we can express $\sigma_{d i s}$ and $\sigma_{b s f}$ in terms of $z$ and $u$. The bound state dissociation rate after taking the thermal averaging into account is

$\left\langle\Gamma_{\eta}^{q^{H}}\right\rangle_{d i s}=g_{g} \frac{4 \pi}{(2 \pi)^{3}} \int_{0}^{\infty} \sigma_{d i s} \frac{E_{B}^{3}\left(1+\frac{u}{z}\right)^{2}}{z\left(e^{z+u}-1\right)} d u$.

The thermally-averaged bound state formation cross section times velocity is given by

$\langle\sigma v\rangle_{b s f}=\int_{0}^{\infty} \sigma_{b s f} \zeta\left(\frac{u}{z}\right)^{1 / 2} \frac{2}{\sqrt{\pi}} u^{1 / 2} e^{-u}$ 


$$
\times\left(1+\frac{1}{e^{z+u}-1}\right) d u .
$$

Finally, the thermally-averaged bound state annihilation decay rate is written as

$$
\left\langle\Gamma_{\eta}^{q^{H}}\right\rangle=\Gamma_{\eta}^{q^{H}} \frac{K_{1}\left(m_{\eta} / T\right)}{K_{2}\left(m_{\eta} / T\right)}
$$

where $m_{\eta}=2 m_{q^{H}}-E_{B}$ and $K_{1,2}\left(m_{\eta} / T\right)$ are the bound state mass and the modified Bessel functions of the second kind, respectively.

Taking both Sommerfeld correction as well as bound state effect into account, we write the thermally-averaged effective annihilation cross section $\left\langle\sigma_{e f f} v\right\rangle$ as

$$
\left\langle\sigma_{e f f} v\right\rangle=\frac{1}{2}\langle\sigma v\rangle_{q^{H} \bar{q}^{H} \rightarrow q \bar{q}, g g} \frac{g_{q^{H}}^{2}(1+\Delta)^{3} e^{-2 \Delta x}}{g_{\text {eff }}^{2}} .
$$

In this expression, we have defined the following parameters

$$
\begin{aligned}
x & \equiv \frac{m_{D}}{T}, \\
\Delta & \equiv \frac{m_{q^{H}}-m_{D}}{m_{D}}, \\
g_{\text {eff }} & \equiv g_{D}+g_{q^{H}}(1+\Delta)^{3 / 2} e^{-\Delta x},
\end{aligned}
$$

where $g_{D}=6$ is the DM degree of freedom. The explicit

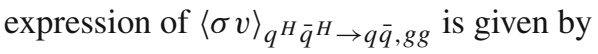

$$
\begin{aligned}
\langle\sigma v\rangle_{q^{H} \bar{q}^{H} \rightarrow q \bar{q}, g g}= & \left\langle\sigma v_{\text {rel }}\left(q^{H} \bar{q}^{H} \rightarrow q \bar{q}\right)\right\rangle_{s, \text { Somm }} \\
& +\left\langle\sigma v_{r e l}\left(q^{H} \bar{q}^{H} \rightarrow g g\right)\right\rangle_{s, \text { Somm }} \\
& +\left\langle\sigma v_{r e l}\left(q^{H} \bar{q}^{H} \rightarrow q \bar{q}\right)\right\rangle_{p} \\
& +\left\langle\sigma v_{r e l}\left(q^{H} \bar{q}^{H} \rightarrow g g\right)\right\rangle_{p} \\
& +\frac{1}{4}\left\langle\sigma v_{r e l}\right\rangle_{b s f} \frac{\left\langle\Gamma_{\eta}^{q^{H}}\right\rangle}{\left\langle\Gamma_{\eta}^{q^{H}}\right\rangle+\frac{1}{4}\left\langle\Gamma_{\eta}^{q^{H}}\right\rangle_{d i s}},
\end{aligned}
$$

where $\left\langle\sigma v_{r e l}\left(q^{H} \bar{q}^{H} \rightarrow q \bar{q}\right)\right\rangle_{p}$ and $\left\langle\sigma v_{r e l}\left(q^{H} \bar{q}^{H} \rightarrow g g\right)\right\rangle_{p}$ stand for the $P$-wave part of the Eqs. (27) and (28). The factor of 1/4 in front of $\left\langle\sigma v_{\text {rel }}\right\rangle_{b s f}$ and $\left\langle\Gamma_{\eta}^{q^{H}}\right\rangle_{d i s}$ is needed since we only consider the $S=0$ bound state. The DM relic abundance can be calculated by [89]

$\Omega_{D} h^{2} \cong \frac{1.07 \times 10^{9} \mathrm{GeV}^{-1}}{J g_{*}^{1 / 2} m_{\mathrm{Pl}}}$,

where $g_{*}$ and $m_{\mathrm{Pl}}$ are the number of massless degree of freedom associated with the energy density and the Planck mass, respectively. The $J$ factor is given by

$J=\int_{x_{F}}^{\infty} \frac{\left\langle\sigma_{e f f} v\right\rangle}{x^{2}} d x$

where $x=m_{D} / T$ and $x_{F}$ is the value of $x$ at freeze-out. However, as Sommerfeld correction as well as bound state effect become important at temperature well below the freeze-out, we set the lower bound of integration to 27. In this work, we take all the 6 heavy quarks $\left(u^{H}, d^{H}, s^{H}, c^{H}, b^{H}\right.$, and $\left.t^{H}\right)$ into account when calculating the Sommerfeld correction as well as bound state effect.

The effects of QCD Sommerfeld correction and QCD bound state on DM relic density are shown in Fig. 7. The gray band in this figure represents the observed DM relic from PLANCK collaboration within $2 \sigma$ significance. The red solid curve comes from the perturbative calculation given in Eqs. (27) and (28). The Sommerfeld correction, as described by the green solid curve, reduces the perturbative cross section due to the cancellation between the Sommerfeld enhancement in $q^{H} \bar{q}^{H} \rightarrow g g$ and the Sommerfeld suppression for $q^{H} \bar{q}^{H} \rightarrow q \bar{q}$ channels [59]. As a result, it increases the DM relic density, shifting the observed relic towards the smaller DM mass.

The vertical dashed lines in Fig. 7 denote the lower bound of DM mass that satisfies the observed relic abundance. The black dashed line is located at $1377 \mathrm{GeV}$. This corresponds to the perturbative calculation. At $1340 \mathrm{GeV}$, the orange dashed line comes from the Sommerfeld correction. This $2.7 \%$ shift demonstrates that the Sommerfeld correction does not significantly affect the DM relic abundance. The bound state effect along with the Sommerfeld correction is shown by the blue solid curve. The lower bound of the DM mass is shifted from 1340 to $1365 \mathrm{GeV}$ as given by the purple dashed line. This shows that the bound state effect, in contrast to the Sommerfeld effect, slightly reduces the DM relic density.

When the electric charge of each $q^{H}$ and $\bar{q}^{H}, Q_{q^{H}}$, is considered, the Coulomb-like potential between them becomes more attractive. This electromagnetic (EM) correction modifies the coupling of the corresponding Coulomb-like potentials as $\zeta \rightarrow \zeta+\alpha_{\mathrm{EM}} Q_{q^{H}}^{2}$ and $\zeta^{\prime} \rightarrow \zeta^{\prime}+\alpha_{\mathrm{EM}} Q_{q^{H}}^{2}$, where $\alpha_{\mathrm{EM}}$ is the EM fine structure constant. All quantities depending on these couplings are changed as well. The EM correction for both Sommerfeld and the bound state effects are shown in Fig. 8. As in Fig. 7, the green (blue) solid curve describes the Sommerfeld (Sommerfeld and the bound state) effect. The black dotted curve is the EM correction of the Sommerfeld effect. This weakens the value of the relic density by $0.19 \%$ at $1340 \mathrm{GeV}$. Furthermore, another similar reduction is also observed when one considers the EM correction of the bound state effect along with the Sommerfeld correction. This is shown by the red dashed curve in Fig. 8. In this case, the reduction of the relic density is about $0.50 \%$ at 
Fig. 7 DM relic density plotted against its mass for three different cases: perturbative calculation (red solid line) without Sommerfeld correction (SC) and bound state formation (BSF), perturbative calculation with SC in $S$-wave (green solid line), and by considering both SC and BSF (blue solid line). The gray band is the observed PLANCK data within $2 \sigma$

Fig. 8 The EM correction for Sommerfeld correction (SC) is given by the black dotted line. The red dashed line represents the EM correction for both SC and bound state effect (BSF).

The gray band is the observed relic density from PLANCK collaboration within $2 \sigma$
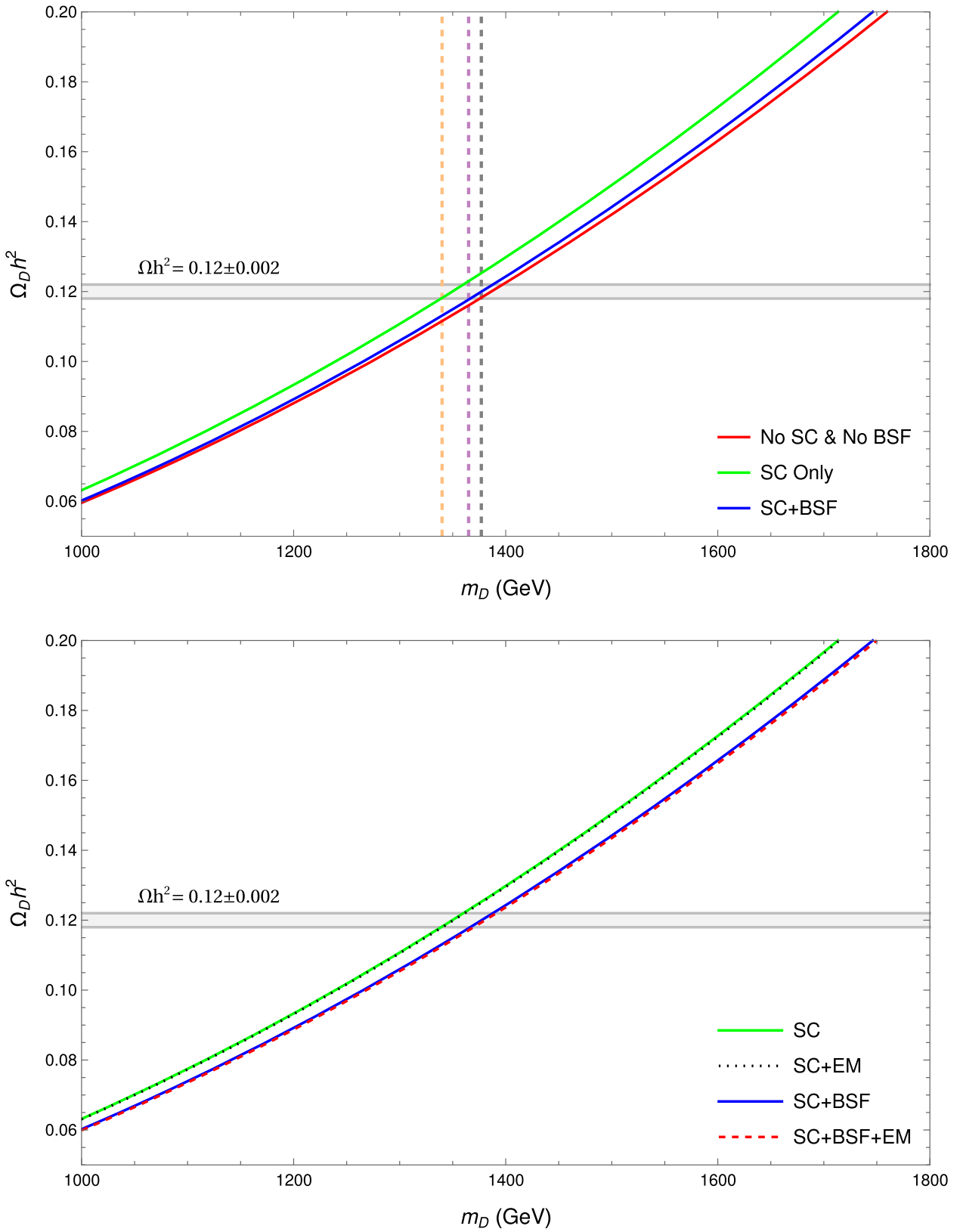

$1365 \mathrm{GeV}$. These results demonstrate that the EM correction is negligibly small and can be omitted in the calculation of the DM relic abundance.

We see that non-perturbative effects such as the QCD Sommerfeld correction as well as the QCD bound state effect do not significantly change the DM relic abundance. Thus, one can safely ignore these effects and carry out the perturbative calculation given by Eqs. (27) and (28).

\section{Constraining parameter space in G2HDM}

We see from the preceding sections that the Goldstone-like DM lives in the limited parameter space region. Moreover, the particular value of $f_{n} / f_{p} \approx-2.00$ is needed to avoid the XENON1T limit. Thus, in this subsection, we focus our discussion on the triplet-like DM when evaluating the surviving parameter space in G2HDM.

We collect the allowed parameter regions from the SGSC+RD+DD constraints in Tables 4 and 5. In this work, the allowed parameter regions are collected in the right column of these tables. The left column lists the surviving parameter space from [32]. As a general remark, we see that the inclusion of heavy fermions put the allowed parameter space into the smaller region.

As discussed in [32], the RD+DD constraints put a strong limits on $\lambda_{\Phi}, \lambda_{H \Delta}$, and $\lambda_{\Phi \Delta}$. This can be seen from their 
Table 4 The surviving parameter space after DM constraints

\begin{tabular}{ll}
\hline Allowed parameter ranges without $f^{H}$ & Allowed parameter ranges with $f^{H}$ \\
\hline $6.35 \times 10^{-3}<\lambda_{\Phi}<4.09$ & $4.24 \times 10^{-3}<\lambda_{\Phi}<3.98$ \\
$-3.39<\lambda_{H \Delta}<4.07$ & $-3.16<\lambda_{H \Delta}<4.16$ \\
$-0.07<\lambda_{\Phi \Delta}<6.62$ & $-0.30<\lambda_{\Phi \Delta}<6.10$ \\
$-5.67<\lambda_{H \Phi}<3.41$ & $-5.33<\lambda_{H \Phi}<2.54$ \\
$-0.01<\lambda_{H \Phi}^{\prime}<15.90$ & $0.01<\lambda_{H \Phi}^{\prime}<14.36$ \\
$1.29 \times 10^{-1}<\lambda_{H}<2.80$ & $1.30 \times 10^{-1}<\lambda_{H}<2.69$ \\
$-22.74<\lambda_{H}^{\prime}<9.57$ & $-21.75<\lambda_{H}^{\prime}<7.61$ \\
$1.01 \times 10^{-4}<\lambda_{\Delta}<4.99$ & $1.20 \times 10^{-4}<\lambda_{\Delta}<4.84$ \\
$7.16 \times 10^{-3}<g_{H}<0.10$ & $3.37 \times 10^{-3}<g_{H}<0.10$ \\
$1.01 \times 10^{-8}<g_{X}<3.55 \times 10^{-2}$ & $1.01 \times 10^{-8}<g_{X}<3.01 \times 10^{-2}$ \\
\hline
\end{tabular}

Table 5 The surviving dimensionful parameter space after DM constraints

\begin{tabular}{ll}
\hline Allowed parameter ranges without $f^{H}($ in GeV) & Allowed parameter ranges with $f^{H}($ in $\mathrm{GeV})$ \\
\hline $2.75<M_{H \Delta}<4.99 \times 10^{3}$ & $-2.44 \times 10^{3}<M_{H \Delta}<4.51 \times 10^{3}$ \\
$0.01<M_{\Phi \Delta}<49.9$ & $0.00<M_{\Phi \Delta}<49.8$ \\
$5.00 \times 10^{2}<v_{\Delta}<2.00 \times 10^{4}$ & $5.07 \times 10^{2}<v_{\Delta}<1.99 \times 10^{4}$ \\
$4.15 \times 10^{4}<v_{\Phi}<1.00 \times 10^{5}$ & $3.99 \times 10^{4}<v_{\Phi}<9.99 \times 10^{4}$ \\
\hline
\end{tabular}

explicit appearance in $D D^{*} h_{j}$ couplings in Eq. (A2). Due to its correlation with $\lambda_{\Phi \Delta}$, these constraints restrict $\lambda_{\Phi}$ as well. As a consequence of the loose requirement that we impose on triplet-like DM, $f_{\Delta_{p}}>2 / 3$, the $G_{H}^{p}$ component becomes non-negligible. In fact, there are points with $f_{G_{H}^{p}}=$ $1 / 3$ in our scan. Consequently, this constrains $\lambda_{H \Phi}$ and $\lambda_{H \Phi}^{\prime}$ which are relevant for the $f_{G_{H}^{p}}$ component as one can see from Eq. (A4). Furthermore, there are indirect constraints on other parameters due to the mixing angles which appear in Eqs. (A2) and (A4).

From previous sections, especially the relic density of triplet-like DM, we see the importance of the $D D^{*} h_{j}$ couplings. In [32], these couplings play important role in the relic density calculation for all DM mass. However, these couplings are relevant only in the $\mathrm{GeV}$ regime (region (i) to (iv)) when heavy fermions are included. This significantly cuts the allowed parameter space of the scalar sector in G2HDM. The same reduction also holds for $g_{H}$ and $g_{X}$. These two gauge couplings correspond to the $Z_{i}$ mediated diagrams, especially for $W^{+} W^{-}$as well as $W_{L}^{+} W_{L}^{-}$final states in region (iii) and (iv) (although these contributions are $P$-wave suppressed). Furthermore, $g_{H}$ and $v_{\Phi}$ receive additional constraints from the lower allowed DM mass as in Eq. (22).

Finally, the constraints from DM physics are not sensitive to limit $v_{\Delta}, M_{\Phi \Delta}$, and $M_{H \Delta}$. In this paper, we extend the scan range for $M_{H \Delta}$ to include the negative values of the parameter space. The motivation is to collect more points in our scan. This explains the broader allowed parameter range for $M_{H \Delta}$ as one can see from Table 5 .

\section{Summary and conclusion}

The motivation of this paper is to study the effects of heavy fermions on the complex scalar DM phenomenology which is omitted in the previous work. Taking the constraints from the observed relic abundance provided by PLANCK collaboration as well as the null result from XENON1T experiment into account, we compare our calculations against these experimental data. By imposing 5\% mass splitting between the DM and heavy fermions as well as lowering their mass to $1 \mathrm{TeV}$, we demonstrate that the heavy fermions significantly affect the complex scalar DM phenomenology. The $\mathrm{DM}$ relic abundance is reduced drastically in the $\mathrm{TeV}$ regime of the DM mass. This happens mainly because of the QCD interactions, $q^{H} \bar{q}^{H} \rightarrow q \bar{q}, g g$.

We further include the QCD Sommerfeld correction as well as the QCD bound state effect in our relic density calculation. We learn that these non-perturbative effects do not change the perturbative calculation in a significant way. On the other hand, heavy fermions weaken the direct search limit given by the XENON1T experiment. This is due to their destructive contribution in the relevant Feynman diagrams. These results put additional constraints on the parameter space of the G2HDM model. This makes the allowed region in the parameter space becomes narrower. We conclude that the inclusion of heavy fermions on the complex scalar DM phenomenology is crucial and can not be neglected.

As an additional remark, the new heavy fermions can be produced by the current collider experiment at the LHC. This search can put a further limit on the mass splitting between 
DM and heavy fermions. The detail study of the properties of new heavy fermions including its production mechanism and the decay modes is beyond the scope of this paper and it will be presented in a separate work in the future.

Acknowledgements We would like to thank Prof. Tzu-Chiang Yuan and Prof. Chuan-Ren Chen who encourage the authors to write this paper. We are grateful to Dr. Raymundo Ramos, Dr. Yue-Lin Sming Tsai, Dr. Van Que Tran, and Dr. Yu-Xiang Lin who provide the important resources for this work. CSN would like to thank Prof. Hsiang-Nan $\mathrm{Li}$ for useful discussions regarding the evaluation of the strong coupling at different scale in QCD bound state effect. A special thanks is addressed to Prof. Martin Spinrath for allowing the authors to do the computation using his machine. BD would like to acknowledge support from Thailand Science Research and Innovation and Suranaree University of Technology through SUT-Ph.D. Scholarship Program for ASEAN.

Data Availability Statement This manuscript has associated data in data repository. [Authors' comment: All data included in this manuscript are available upon reasonable request by contacting with the corresponding author.]

Open Access This article is licensed under a Creative Commons Attribution 4.0 International License, which permits use, sharing, adaptation, distribution and reproduction in any medium or format, as long as you give appropriate credit to the original author(s) and the source, provide a link to the Creative Commons licence, and indicate if changes were made. The images or other third party material in this article are included in the article's Creative Commons licence, unless indicated otherwise in a credit line to the material. If material is not included in the article's Creative Commons licence and your intended use is not permitted by statutory regulation or exceeds the permitted use, you will need to obtain permission directly from the copyright holder. To view a copy of this licence, visit http://creativecomm ons.org/licenses/by/4.0/.

Funded by SCOAP ${ }^{3}$.

\section{Appendix A: Feynman rules}

Here we collect the most important couplings to the DM analysis in various interactions as discussed in the text. The gauge couplings $g_{H}$ and $g_{X}$ are related to $S U(2)_{H}$ and $U(1)_{X}$ gauge group, respectively. For the scalar-scalar-gauge derivative vertices, we use the convention that all momenta are incoming.

Dominant couplings for triplet-like DM

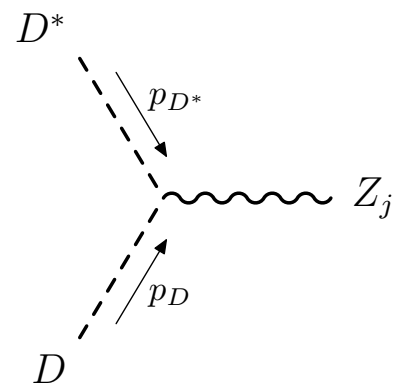

$$
\approx i g_{H}\left(\mathcal{O}_{32}^{D}\right)^{2} \mathcal{O}_{2 j}^{G}\left(p_{D^{*}}-p_{D}\right)_{\mu}
$$

$D^{*}$

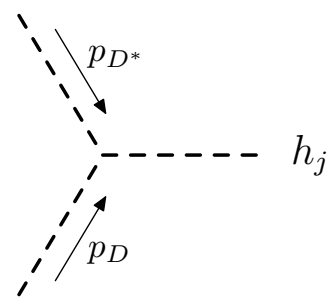

$D$

$$
\begin{aligned}
\approx & i\left[-\lambda_{H \Delta} v \mathcal{O}_{1 j}\right. \\
& \left.-\lambda_{\Phi \Delta} v_{\Phi} \mathcal{O}_{2 j}+2 \lambda_{\Delta} v_{\Delta} \mathcal{O}_{3 j}\right]\left(\mathcal{O}_{32}^{D}\right)^{2}
\end{aligned}
$$

Dominant couplings for Goldstone boson-like DM
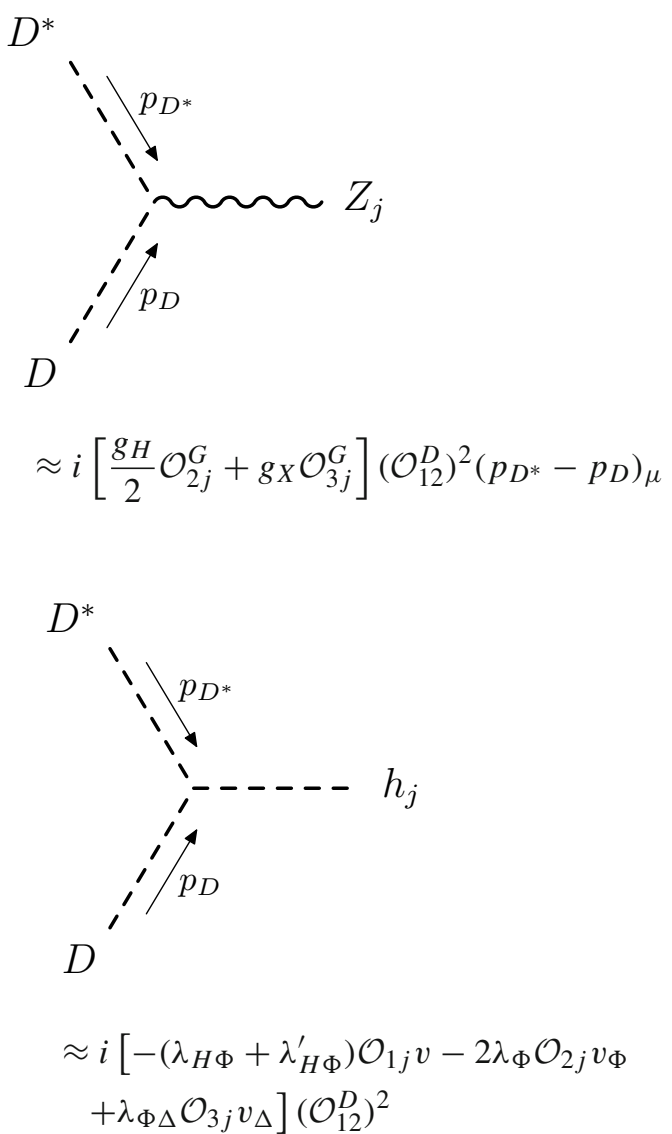


\section{References}

1. G.C. Branco, P.M. Ferreira, L. Lavoura, M.N. Rebelo, M. Sher, J.P. Silva, Phys. Rep. 516, 1 (2012). https://doi.org/10.1016/j.physrep. 2012.02.002 arXiv:1106.0034 [hep-ph]

2. H.E. Logan, arXiv:1406.1786 [hep-ph]

3. N.G. Deshpande, E. Ma, Phys. Rev. D 18, 2574 (1978). https://doi. org/10.1103/PhysRevD.18.2574

4. L. Lopez Honorez, E. Nezri, J.F. Oliver, M.H.G. Tytgat, JCAP 0702, 028 (2007). https://doi.org/10.1088/1475-7516/2007/02/ 028 arXiv:hep-ph/0612275

5. C. Arina, F.S. Ling, M.H.G. Tytgat, JCAP 0910, 018 (2009). https:// doi.org/10.1088/1475-7516/2009/10/018 arXiv:0907.0430 [hep$\mathrm{ph}]$

6. E. Nezri, M.H.G. Tytgat, G. Vertongen, JCAP 0904, 014 (2009). https://doi.org/10.1088/1475-7516/2009/04/014 arXiv:0901.2556 [hep-ph]

7. X. Miao, S. Su, B. Thomas, Phys. Rev. D 82, 035009 (2010). https:// doi.org/10.1103/PhysRevD.82.035009 arXiv:1005.0090 [hep-ph]

8. M. Gustafsson, S. Rydbeck, L. Lopez-Honorez, E. Lundstrom, Phys. Rev. D 86, 075019 (2012). https://doi.org/10.1103/ PhysRevD.86.075019 arXiv:1206.6316 [hep-ph]

9. A. Arhrib, R. Benbrik, N. Gaur, Phys. Rev. D 85, 095021 (2012). https://doi.org/10.1103/PhysRevD.85.095021 arXiv:1201.2644 [hep-ph]

10. A. Arhrib, R. Benbrik, T.C. Yuan, Eur. Phys. J. C 74, 2892 (2014). https://doi.org/10.1140/epjc/s10052-014-2892-5 arXiv:1401.6698 [hep-ph]

11. B. Swiezewska, M. Krawczyk, Phys. Rev. D 88(3), 035019 (2013). https://doi.org/10.1103/PhysRevD.88.035019 arXiv:1212.4100 [hep-ph]

12. A. Arhrib, Y.L.S. Tsai, Q. Yuan, T.C. Yuan, JCAP 1406, 030 (2014). https://doi.org/10.1088/1475-7516/2014/06/030 arXiv: 1310.0358 [hep-ph]

13. A. Goudelis, B. Herrmann, O. Stål, JHEP 1309, 106 (2013). https:// doi.org/10.1007/JHEP09(2013)106 arXiv:1303.3010 [hep-ph]

14. M. Krawczyk, D. Sokolowska, P. Swaczyna, B. Swiezewska, JHEP 1309, 055 (2013). https://doi.org/10.1007/JHEP09(2013)055 arXiv:1305.6266 [hep-ph]

15. M. Krawczyk, D. Sokolowska, P. Swaczyna, B. Swiezewska, Acta Phys. Polon. B 44(11), 2163 (2013). https://doi.org/10.5506/ APhysPolB.44.2163 arXiv:1309.7880 [hep-ph]

16. A. Ilnicka, M. Krawczyk, T. Robens, Phys. Rev. D 93(5), 055026 (2016). https://doi.org/10.1103/PhysRevD.93.055026 arXiv:1508.01671 [hep-ph]

17. M.A. Diaz, B. Koch, S. Urrutia-Quiroga, Adv. High Energy Phys. 2016, 8278375 (2016). https://doi.org/10.1155/2016/8278375 arXiv:1511.04429 [hep-ph]

18. K.P. Modak, D. Majumdar, Astrophys. J. Suppl. 219(2), 37 (2015). https://doi.org/10.1088/0067-0049/219/2/37 arXiv:1502.05682 [hep-ph]

19. T.W. Kephart, T.C. Yuan, Nucl. Phys. B 906, 549 (2016). https:// doi.org/10.1016/j.nuclphysb.2016.03.023 arXiv:1508.00673 [hep$\mathrm{ph}]$

20. F.S. Queiroz, C.E. Yaguna, JCAP 1602(02), 038 (2016). https://doi. org/10.1088/1475-7516/2016/02/038 arXiv:1511.05967 [hep-ph]

21. C. Garcia-Cely, M. Gustafsson, A. Ibarra, JCAP 1602(02), 043 (2016). https://doi.org/10.1088/1475-7516/2016/02/043 arXiv:1512.02801 [hep-ph]

22. M. Hashemi, S. Najjari, Eur. Phys. J. C 77(9), 592 (2017). https:// doi.org/10.1140/epjc/s10052-017-5159-0 arXiv:1611.07827 [hep-ph]

23. P. Poulose, S. Sahoo, K. Sridhar, Phys. Lett. B 765, 300 (2017). https://doi.org/10.1016/j.physletb.2016.12.022 arXiv:1604.03045 [hep-ph]
24. A. Alves, D.A. Camargo, A.G. Dias, R. Longas, C.C. Nishi, F.S. Queiroz, JHEP 1610, 015 (2016). https://doi.org/10.1007/ JHEP10(2016)015 arXiv:1606.07086 [hep-ph]

25. A. Datta, N. Ganguly, N. Khan, S. Rakshit, Phys. Rev. D 95(1), 015017 (2017). https://doi.org/10.1103/PhysRevD.95. 015017 arXiv: 1610.00648 [hep-ph]

26. A. Belyaev, G. Cacciapaglia, I.P. Ivanov, F. Rojas-Abatte, M. Thomas, Phys. Rev. D 97(3), 035011 (2018). https://doi.org/10. 1103/PhysRevD.97.035011 arXiv:1612.00511 [hep-ph]

27. A. Belyaev, T.R. FernandezPerezTomei, P.G. Mercadante, C.S. Moon, S. Moretti, S.F. Novaes, L. Panizzi, F. Rojas, M. Thomas, Phys. Rev. D 99(1), 015011 (2019). https://doi.org/10.1103/ PhysRevD.99.015011 arXiv:1809.00933 [hep-ph]

28. S. Banerjee, F. Boudjema, N. Chakrabarty, G. Chalons, H. Sun, Phys. Rev. D 100(9), 095024 (2019). https://doi.org/10.1103/ PhysRevD.100.095024 arXiv:1906.11269 [hep-ph]

29. S. Banerjee, F. Boudjema, N. Chakrabarty, H. Sun, Phys. Rev. D 104, 075002 (2021). https://doi.org/10.1103/PhysRevD.104. 075002 arXiv:2101.02165 [hep-ph]

30. S. Biondini, M. Laine, JHEP 08, 047 (2017). https://doi.org/10. 1007/JHEP08(2017)047 arXiv:1706.01894 [hep-ph]

31. W.C. Huang, Y.L.S. Tsai, T.C. Yuan, JHEP 1604, 019 (2016). https://doi.org/10.1007/JHEP04(2016)019 arXiv:1512.00229 [hep-ph]

32. C.R. Chen, Y.X. Lin, C.S. Nugroho, R. Ramos, Y.L.S. Tsai, T.C. Yuan, Phys. Rev. D 101(3), 035037 (2020). https://doi.org/10. 1103/PhysRevD.101.035037 arXiv:1910.13138 [hep-ph]

33. W.C. Huang, Y.L.S. Tsai, T.C. Yuan, Nucl. Phys. B 909, 122 (2016). https://doi.org/10.1016/j.nuclphysb.2016.05.002 arXiv:1512.07268 [hep-ph]

34. W.C. Huang, H. Ishida, C.T. Lu, Y.L.S. Tsai, T.C. Yuan, Eur. Phys. J. C 78(8), 613 (2018). https://doi.org/10.1140/epjc/ s10052-018-6067-7 arXiv:1708.02355 [hep-ph]

35. C.R. Chen, Y.X. Lin, V.Q. Tran, T.C. Yuan, Phys. Rev. D 99(7), 075027 (2019). https://doi.org/10.1103/PhysRevD.99. 075027 arXiv: 1810.04837 [hep-ph]

36. A. Arhrib, W.C. Huang, R. Ramos, Y.L.S. Tsai, T.C. Yuan, Phys. Rev. D 98(9), 095006 (2018). https://doi.org/10.1103/PhysRevD. 98.095006 arXiv:1806.05632 [hep-ph]

37. C.T. Huang, R. Ramos, V.Q. Tran, Y.L.S. Tsai, T.C. Yuan, JHEP 1909, 048 (2019). https://doi.org/10.1007/JHEP09(2019)048 arXiv:1905.02396 [hep-ph]

38. R.N. Mohapatra, G. Senjanovic, Phys. Rev. Lett. 44, 912 (1980). https://doi.org/10.1103/PhysRevLett.44.912

39. W.Y. Keung, G. Senjanovic, Phys. Rev. Lett. 50, 1427 (1983). https://doi.org/10.1103/PhysRevLett.50.1427

40. P.Q. Hung, Phys. Lett. B 649, 275 (2007). https://doi.org/10.1016/ j.physletb.2007.03.067. arXiv:hep-ph/0612004

41. P.Q. Hung, T. Le, V.Q. Tran, T.C. Yuan, Nucl. Phys. B 932, 471 (2018). https://doi.org/10.1016/j.nuclphysb.2018.05.020 arXiv:1701.01761 [hep-ph]

42. C.F. Chang, C.H.V. Chang, C.S. Nugroho, T.C. Yuan, Nucl. Phys. B 910, 293-308 (2016). https://doi.org/10.1016/j.nuclphysb.2016. 07.009 arXiv:1602.00680 [hep-ph]

43. P.Q. Hung, T. Le, V.Q. Tran, T.C. Yuan, JHEP 1512, 169 (2015). https://doi.org/10.1007/JHEP12(2015)169 arXiv:1508.07016 [hep-ph]

44. C.F. Chang, P.Q. Hung, C.S. Nugroho, V. Tran, T.C. Yuan, Nucl. Phys. B 928, 21-37 (2018). https://doi.org/10.1016/j.nuclphysb. 2018.01.007 arXiv:1702.04516 [hep-ph]

45. P.Q. Hung, Y.X. Lin, C.S. Nugroho, T.C. Yuan, Nucl. Phys. B 927, 166-183 (2018). https://doi.org/10.1016/j.nuclphysb.2017.12.014 arXiv:1709.01690 [hep-ph]

46. P. Ko, Y. Omura, C. Yu, Phys. Lett. B 717, 202 (2012). https://doi. org/10.1016/j.physletb.2012.09.019 arXiv:1204.4588 [hep-ph] 
47. M.D. Campos, D. Cogollo, M. Lindner, T. Melo, F.S. Queiroz, W. Rodejohann, JHEP 1708, 092 (2017). https://doi.org/10.1007/ JHEP08(2017)092 arXiv:1705.05388 [hep-ph]

48. D.A. Camargo, L. Delle Rose, S. Moretti, F.S. Queiroz, Phys. Lett. B 793, 150 (2019). https://doi.org/10.1016/j.physletb.2019.04.048 arXiv: 1805.08231 [hep-ph]

49. D.A. Camargo, A.G. Dias, T.B. de Melo, F.S. Queiroz, JHEP 1904, 129 (2019). https://doi.org/10.1007/JHEP04(2019)129 arXiv: 1811.05488 [hep-ph]

50. D.A. Camargo, M.D. Campos, T.B. de Melo, F.S. Queiroz, Phys. Lett. B 795, 319 (2019). https://doi.org/10.1016/j.physletb.2019. 06.020 arXiv:1901.05476 [hep-ph]

51. D. Cogollo, R.D. Matheus, T.B. de Melo, F.S. Queiroz, Phys. Lett. B 797, 134813 (2019). https://doi.org/10.1016/j.physletb. 2019.134813 arXiv:1904.07883 [hep-ph]

52. http://pdg.arsip.lipi.go.id/2017/reviews/rpp2016-rev-susy-2experiment.pdf

53. S.L. Glashow, S. Weinberg, Phys. Rev. D 15, 1958 (1977). https:// doi.org/10.1103/PhysRevD.15.1958

54. E.A. Paschos, Phys. Rev. D 15, 1966 (1977). https://doi.org/10. 1103/PhysRevD.15.1966

55. N. Aghanim et al., [Planck Collaboration], arXiv:1807.06209 [astro-ph.CO]

56. E. Aprile et al., [XENON Collaboration], Phys. Rev. Lett. 121(11), 111302 (2018). https://doi.org/10.1103/PhysRevLett.121.111302. arXiv:1805.12562 [astro-ph.CO]

57. G. Belanger, F. Boudjema, A. Goudelis, A. Pukhov, B. Zaldivar, Comput. Phys. Commun. 231, 173 (2018). https://doi.org/10.1016/ j.cpc.2018.04.027 arXiv:1801.03509 [hep-ph]

58. G. Belanger, F. Boudjema, A. Pukhov, A. Semenov, Comput. Phys. Commun. 180, 747 (2009). https://doi.org/10.1016/j.cpc.2008.11. 019 arXiv:0803.2360 [hep-ph]

59. S. El Hedri, A. Kaminska, M. de Vries, J. Zurita, JHEP 04, 118 (2017). https://doi.org/10.1007/JHEP04(2017)118 arXiv:1703.00452 [hep-ph]

60. A. Sommerfeld, Ann. Phys. 403, 257 (1931)

61. J.L. Feng, M. Kaplinghat, H. Tu, H.B. Yu, JCAP 07, 004 (2009). https://doi.org/10.1088/1475-7516/2009/07/004 arXiv:0905.3039 [hep-ph]

62. B. von Harling, K. Petraki, JCAP 12, 033 (2014). https://doi.org/ 10.1088/1475-7516/2014/12/033 arXiv:1407.7874 [hep-ph]

63. K. Petraki, M. Postma, M. Wiechers, JHEP 06, 128 (2015). https:// doi.org/10.1007/JHEP06(2015)128 arXiv:1505.00109 [hep-ph]

64. S. Kim, M. Laine, JHEP 07, 143 (2016). https://doi.org/10.1007/ JHEP07(2016)143 arXiv:1602.08105 [hep-ph]

65. S. Kim, M. Laine, JCAP 01, 013 (2017). https://doi.org/10.1088/ 1475-7516/2017/01/013 arXiv:1609.00474 [hep-ph]

66. K. Petraki, M. Postma, J. de Vries, JHEP 04, 077 (2017). https:// doi.org/10.1007/JHEP04(2017)077 arXiv:1611.01394 [hep-ph]

67. K. Petraki, L. Pearce, A. Kusenko, JCAP 07, 039 (2014). https:// doi.org/10.1088/1475-7516/2014/07/039 arXiv:1403.1077 [hep$\mathrm{ph}]$

68. W. Detmold, M. McCullough, A. Pochinsky, Phys. Rev. D 90(11), 115013 (2014). https://doi.org/10.1103/PhysRevD.90. 115013 arXiv:1406.2276 [hep-ph]
69. H. An, M.B. Wise, Y. Zhang, Phys. Rev. D 93(11), 115020 (2016). https://doi.org/10.1103/PhysRevD.93.115020 arXiv:1604.01776 [hep-ph]

70. C. Kouvaris, K. Langæble, N.G. Nielsen, JCAP 10, 012 (2016). https://doi.org/10.1088/1475-7516/2016/10/012 arXiv:1607.00374 [hep-ph]

71. J. Ellis, F. Luo, K.A. Olive, JHEP 09, 127 (2015). https://doi.org/ 10.1007/JHEP09(2015)127 arXiv:1503.07142 [hep-ph]

72. J. Ellis, J.L. Evans, F. Luo, K.A. Olive, JHEP 02, 071 (2016). https://doi.org/10.1007/JHEP02(2016)071 arXiv:1510.03498 [hep-ph]

73. N. Nagata, H. Otono, S. Shirai, Phys. Lett. B 748, 24-29 (2015). https://doi.org/10.1016/j.physletb.2015.06.044 arXiv:1504.00504 [hep-ph]

74. J.L. Feng, M. Kaplinghat, H.B. Yu, Phys. Rev. D 82, 083525 (2010). https://doi.org/10.1103/PhysRevD.82.083525 arXiv:1005.4678 [hep-ph]

75. H. Iminniyaz, M. Kakizaki, Nucl. Phys. B 851, 57-65 (2011). https://doi.org/10.1016/j.nuclphysb.2011.05.009 arXiv:1008.2905 [astro-ph.CO]

76. A. Hryczuk, R. Iengo, P. Ullio, JHEP 03, 069 (2011). https://doi. org/10.1007/JHEP03(2011)069 arXiv:1010.2172 [hep-ph]

77. A. Mitridate, M. Redi, J. Smirnov, A. Strumia, JCAP 05, 006 (2017). https://doi.org/10.1088/1475-7516/2017/05/006 arXiv:1702.01141 [hep-ph]

78. J. Harz, K. Petraki, JHEP 07, 096 (2018). https://doi.org/10.1007/ JHEP07(2018)096 arXiv:1805.01200 [hep-ph]

79. T. Binder, L. Covi, K. Mukaida, Phys. Rev. D 98(11), 115023 (2018). https://doi.org/10.1103/PhysRevD.98.115023 arXiv:1808.06472 [hep-ph]

80. S. Biondini, S. Vogl, JHEP 02, 016 (2019). https://doi.org/10.1007/ JHEP02(2019)016 arXiv:1811.02581 [hep-ph]

81. N. Brambilla, M.A. Escobedo, J. Ghiglieri, A. Vairo, JHEP 12, 116 (2011). https://doi.org/10.1007/JHEP12(2011)116 arXiv:1109.5826 [hep-ph]

82. S. Biondini, S. Vogl, JHEP 11, 147 (2019). https://doi.org/10.1007/ JHEP11(2019)147 arXiv:1907.05766 [hep-ph]

83. A. De Simone, G.F. Giudice, A. Strumia, JHEP 06, 081 (2014). https://doi.org/10.1007/JHEP06(2014)081 arXiv:1402.6287 [hep$\mathrm{ph}]$

84. W.Y. Keung, I. Low, Y. Zhang, Phys. Rev. D 96(1), 015008 (2017). https://doi.org/10.1103/PhysRevD.96.015008 arXiv:1703.02977 [hep-ph]

85. S.P. Liew, F. Luo, JHEP 02, 091 (2017). https://doi.org/10.1007/ JHEP02(2017)091 arXiv:1611.08133 [hep-ph]

86. M. Srednicki, R. Watkins, K.A. Olive, Nucl. Phys. B 310, 693 (1988). https://doi.org/10.1016/0550-3213(88)90099-5

87. J.R. Ellis, T. Falk, K.A. Olive, M. Srednicki, Astropart. Phys. 13, 181-213 (2000) [Erratum: Astropart. Phys. 15, 413414 (2001)]. https://doi.org/10.1016/S0927-6505(99)00104-8. arXiv:hep-ph/9905481

88. D. Kahawala, Y. Kats, JHEP 09, 099 (2011). https://doi.org/10. 1007/JHEP09(2011)099 arXiv:1103.3503 [hep-ph]

89. A. Berlin, D. Hooper, S.D. McDermott, Phys. Rev. D 89(11), 115022 (2014). https://doi.org/10.1103/PhysRevD.89. 115022 arXiv:1404.0022 [hep-ph] 